\title{
Fabrication and characterization of submicron polymer waveguides by micro-transfer molding
}

\author{
by \\ Te-Wei Wu \\ A thesis submitted to the graduate faculty \\ in partial fulfillment of the requirements for the degree of \\ MASTER OF SCIENCE
Major: Materials Science and Engineering
Program of Study Committee:
Kristen P. Constant, Major Professor
Kai-Ming Ho
Zhiqun Lin

Iowa State University

Ames, Iowa

2009

Copyright $\odot$ Te-Wei Wu, 2009. All rights reserved 


\section{TABLE OF CONTENTS}

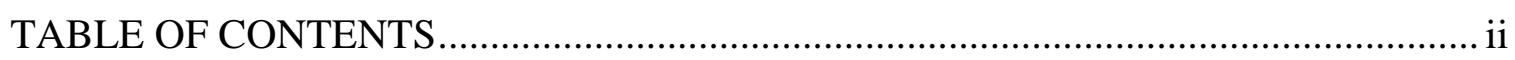

TABLE OF FIGURES .................................................................................... iv

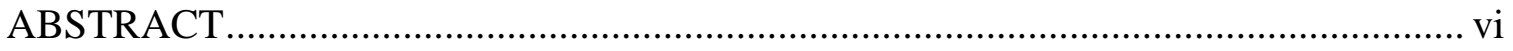

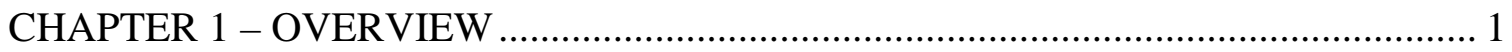

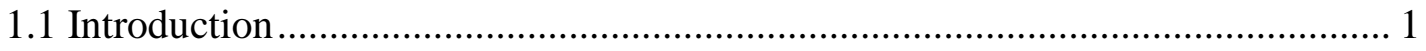

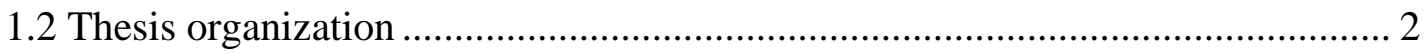

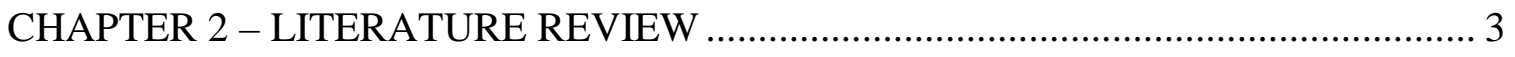

2.1 Previous development of the waveguide structure ....................................... 3

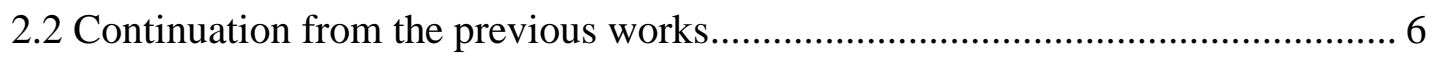

CHAPTER 3 - METHODS AND PROCEDURES ….............................................. 7

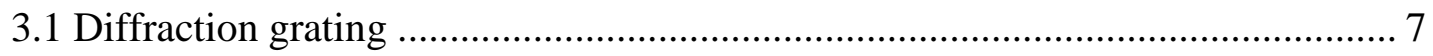

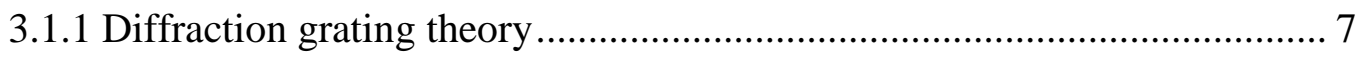

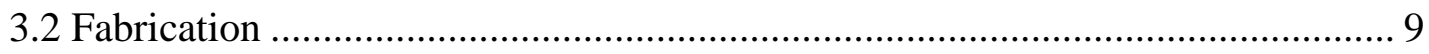

3.2.1 Two-polymer microtransfer molding .............................................. 9

3.2.2 Coupler waveguide by two-polymer microtransfer molding .................. 13

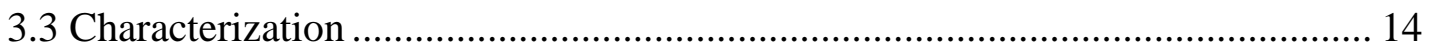

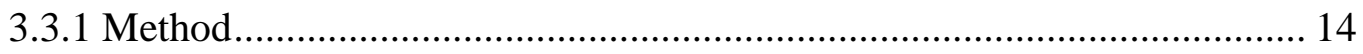

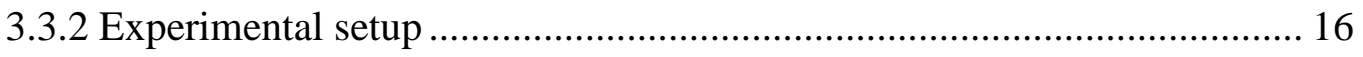

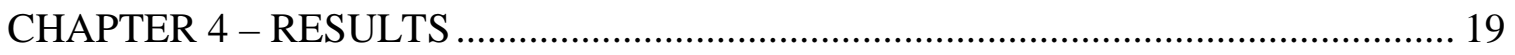

4.1 Waveguide Structures from Scanning Electron Microscope ........................... 19

4.2 Waveguide characterization using a laser.............................................. 21

4.2.1 Experimental setup and theoretical calculation ................................ 21

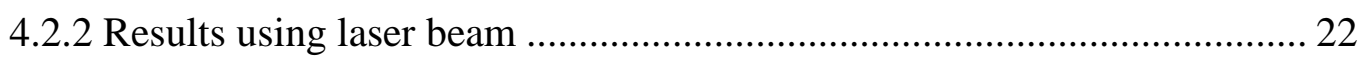


4.3 Waveguide characterization using a white light source ................................. 25

4.3.1 Theoretical calculation and concepts............................................... 25

4.3.2 Results using white light source ....................................................... 30

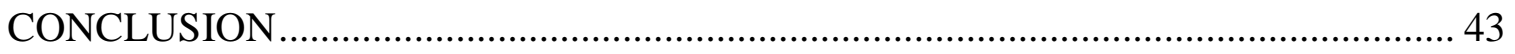

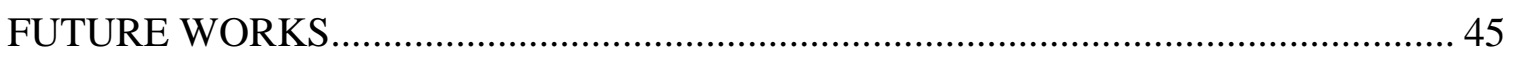

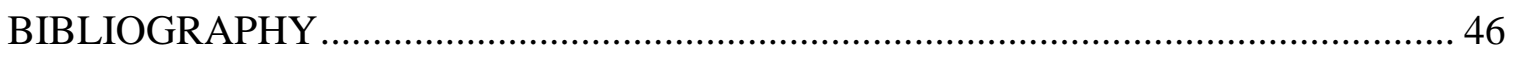




\section{TABLE OF FIGURES}

Figure 1 Schematic diagram of a quantum dot on the waveguide structure.................. 4

Figure 2 Fabricated waveguides array with coupled light sources. (a) SEM micrographs of the waveguides and (b) optical micrograph of the coupled light sources are shown with (c) a schematic illustration of guided light.

Figure 3 SEM images of (a) top and (b) diagonal views of grating coupler. (c) Laser shined on the coupler region and guided by the waveguide. ..................................... 5

Figure 4 Illustration of the theory of diffraction for a planar wavefront. .................... 8

Figure 5 Two polymer microtransfer molding fabrication process. .......................... 11

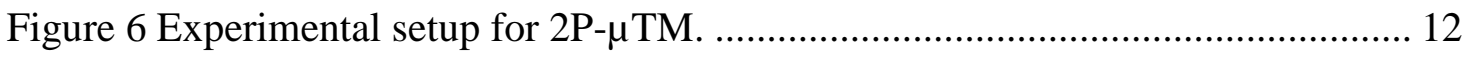

Figure 7 Schematic diagram of the coupler waveguide structure............................. 14

Figure 8 Conceptual representation of the function of the waveguide structure......... 15

Figure 9 Schematic diagram of the experimental setup for characterizing the waveguide.

Figure 10 Schematic diagram showing the placement of the focused light spot on the coupler waveguide.

Figure 11 SEM image of the $2.5 \mu \mathrm{m}$ period waveguide structure ............................... 19

Figure 12 SEM image of the edge of the light coupler........................................... 20

Figure 13 Cross section and dimensions of the waveguides. .................................... 20

Figure 14 Experimental setup to observe the diffraction pattern with a normal incident laser beam.

Figure 15 (a) The diffraction pattern from the coupler is imposed on the paper screen.

(b) The distance from the incident beam to the 1 st order diffraction is measured....... 23

Figure 16 Measured distances to calculate diffraction angle.

Figure 17 Optical image of the guided 1st order diffraction at the end of the waveguide.

Figure 18 The calculated incident angles for multi-order diffraction in the visible range. 
Figure 19 Broadening of peak spectrum due to convergent incident light................. 28

Figure 20 Convergent lens effect of peak broadening for each selected wavelength... 30

Figure 21 Spectrum of the halogen lamp light source............................................. 31

Figure 22 Movement of the light spot from within to out of the coupler................... 32

Figure 23 Optical images of the guided light with varying light spot position........... 33

Figure 24 Spectra of the different position of the light spot on the coupler................ 34

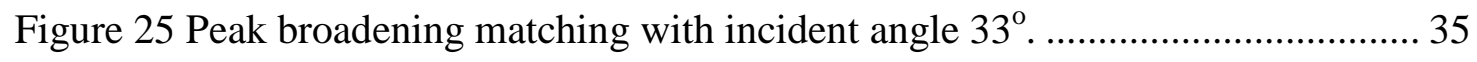

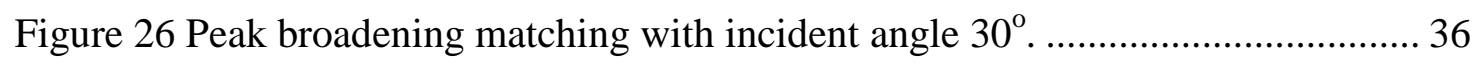

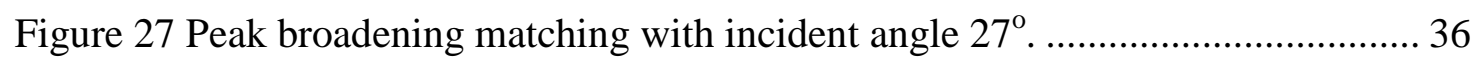

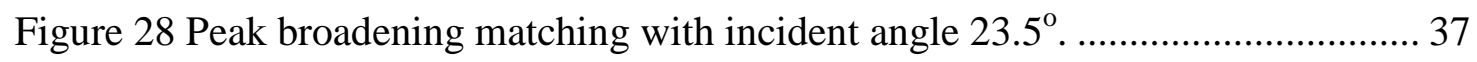

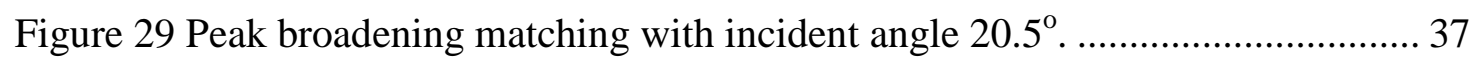

Figure 30 Comparison of the spectra between different incident angles..................... 38

Figure 31 Optical images of the guided light with incident angles of $33^{\circ}(\sim 450 \mathrm{~nm})$,

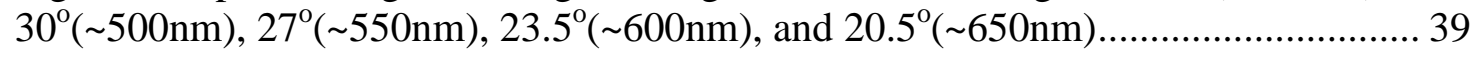

Figure 32 Lorentz fitting for each spectrum using Origin(c) software...................... 40

Figure 33 Comparison between the theoretical and experimental results of the peak

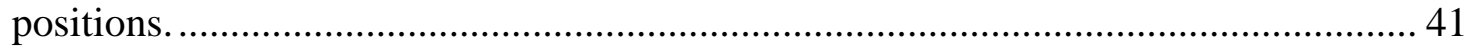

Figure 34 Normalized spectra to the white light source at different incident angles. .. 42 


\section{ABSTRACT}

Various methods exist for fabrication of micron and submicron sized waveguide structures. However, most of them include expensive and time consuming semiconductor fabrication techniques. An economical method for fabricating waveguide structures is introduced and demonstrated in this thesis. This method is established based on previously well-developed photonic crystal fabrication method called two-polymer microtransfer molding. The waveguide in this work functions by a coupler structure that diffracts the incident light into submicron polymer rods. The light is then guided through the rods. Characterization is done by collecting the light that has been guided through the waveguide and exits the end of these submicron polymer bars. The coupling and waveguiding capabilities are demonstrated using two light sources, a laser and white light. 


\section{CHAPTER 1 - OVERVIEW}

\subsection{Introduction}

The concept of waveguides is well established. The most commonly known and used waveguide is the optical fiber which transmits light by total internal reflection. The technology and materials used are well-developed. However, this type of fiber optics is considered to be most useful on the macroscopic scale. Micron and submicron waveguide fabrication usually requires the more expensive and slow processes of photolithography, including deposition and etching. The optical waveguide structure introduced in this thesis uses polymeric materials and a simple, economical fabrication technique. Previously reported fabrication methods for making polymer waveguides include photolithography, dry etching, laser direct writing, and imprinting. ${ }^{1}$ A novel and economical method for fabricating waveguides is developed and introduced in this thesis called two-polymer microtransfer molding $(2 \mathrm{P}-\mu \mathrm{mTM})$. This method provides a much less expensive, relatively fast, and safe way to fabricate the waveguide structure. There are no hazardous chemicals or processes used in this method. $2 \mathrm{P}-\mu \mathrm{mTM}$ can also be used to fabricate three dimensional layer-by-layer structures with high yield and good quality. ${ }^{2}$ This method also suggests the potential for mass production of highly ordered waveguide structures.

Due to their low cost and flexibility, polymeric waveguides have enormous potential applications such as biochemical and chemical sensing, lab on-chip designs, circuit interconnects, and printed board waveguides. ${ }^{1,3,4}$ Also, the wide selection of the 
polymer materials that can be used as a waveguide structure can address the compatibility issues in biosensor materials. ${ }^{5}$

\subsection{Thesis organization}

Chapter 1 introduces the concept and inspiration of the waveguide structure investigated in this thesis, and the motivation for the research. Chapter 2 is a literature review of published work related to different waveguide concepts and structures previously studied by others. Chapter 3 first begins with an explanation of the fundamental concepts of a diffraction grating and then introduces the principal fabrication techniques used as well as the procedures to build the waveguide structure. Finally, Chapter 3 explains the experimental setup for the characterization of the polymer waveguide. Chapter 4 presents the results of waveguide characterization using a laser source and a white light source. 


\section{CHAPTER 2 - LITERATURE REVIEW}

This chapter introduces some previous work on polymer waveguides. The fabrication technique is established, and some characteristics of the waveguide structure and its guiding capabilities are discussed.

\subsection{Previous development of the waveguide structure}

Photonic waveguides have become increasingly important in areas including integrated circuit designs and bio-sensors and applications. Miniaturization of the devices plays an important role in these applications. Polymers have been developed as promising materials for waveguide applications due to their extensive range of properties and adaptable fabrication methods.

One type of photonic device that has been made is composed of waveguides, and

light sources. ${ }^{2}$ Conventional photolithography techniques are commonly used to fabricate waveguide structures. However, the processes are costly and time-consuming. An new fabrication method for this device has been developed based on soft lithography and is called the two-polymer microtransfer molding $(2 \mathrm{P}-\mu \mathrm{TM}) .{ }^{2}$ This introduces a novel and economical way of fabricating a two or three dimensional waveguide structures. Instead of using potentially hazardous methods such deposition and etching, the new method uses non-hazardous polymers and safe procedures to fabricate the waveguide structure.

One of the approaches to introduce a light source is to incorporate an on-chip design using quantum dots as shown in Figure 1. The embedded quantum dots will emit 
light upon excitation by a focused Argon ion laser $(\lambda=365 \mathrm{~nm}){ }^{2}$ The light will then be guided through the waveguides and collected at the end of the waveguides.

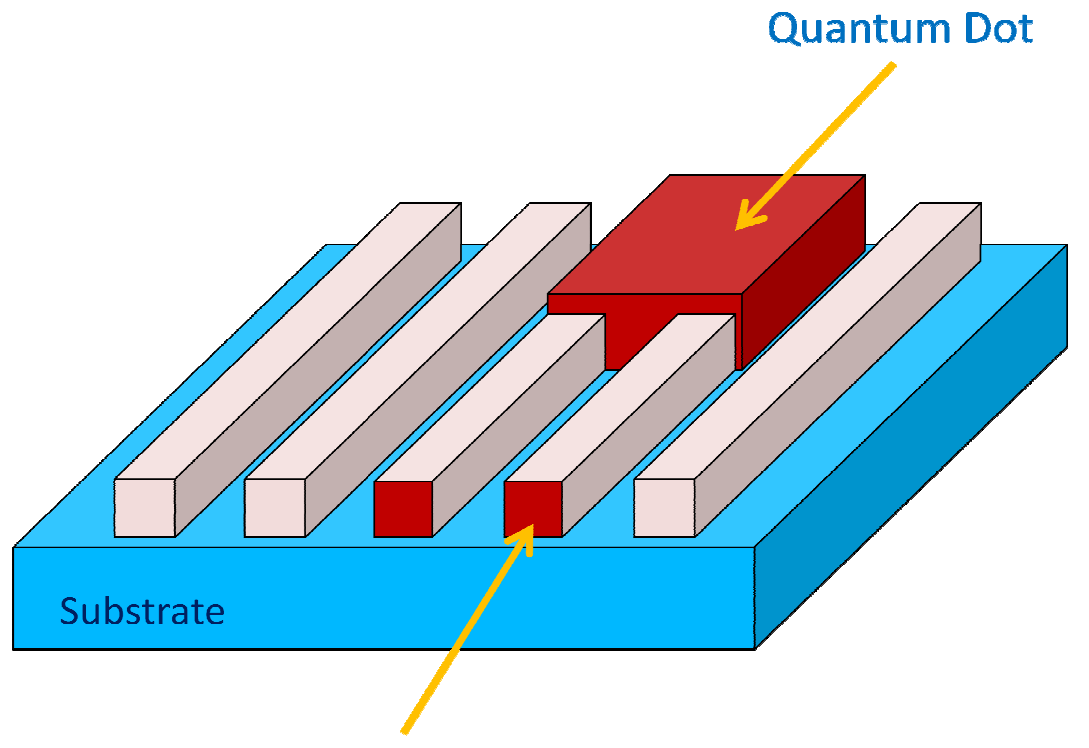

\section{Guided Light}

Figure 1 Schematic diagram of a quantum dot on the waveguide structure.

SEM and optical images are shown for this waveguide structure with embedded quantum dots in Figure 2.

(a)

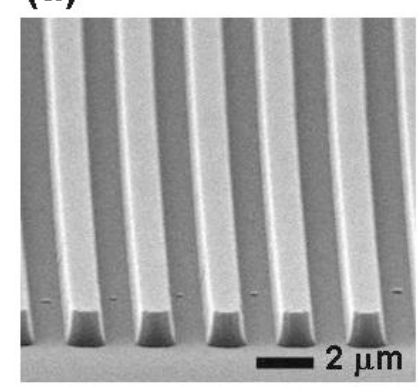

(b)

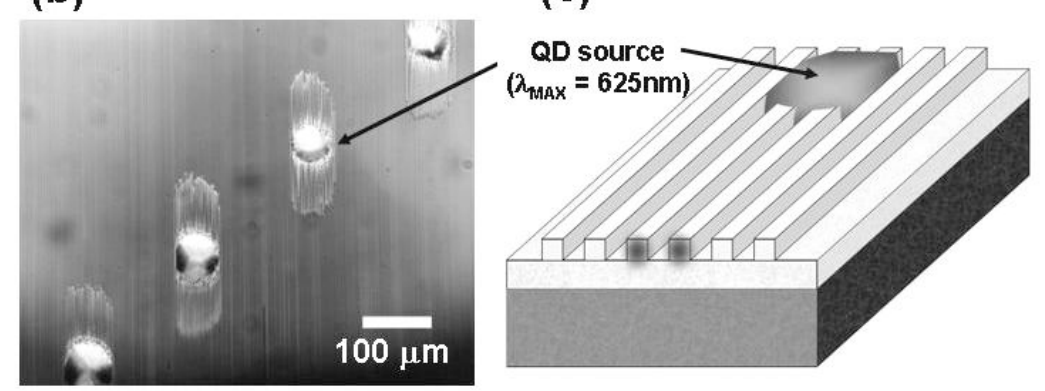

Figure 2 Fabricated waveguides array with coupled light sources. (a) SEM micrographs of the waveguides and (b) optical micrograph of the coupled light sources are shown with (c) a schematic illustration of guided light. ${ }^{2}$ 
Another configuration of a waveguide is to introduce a coupler on the waveguides rather than embed quantum dots. The introduction of the coupler avoids the use of an onchip design of light source. Instead, an external light source can illuminate the coupler. This provides more possibilities as a sensing device. Two waveguides with different periodicities are overlapped $90^{\circ}$ to each other as shown in Figure 3 (a) and (b). ${ }^{2}$ The top pattern has a periodicity of $1 \mu \mathrm{m}$ and the bottom, $2.5 \mu \mathrm{m}$. The overlapping region on the structure acts to couple the incident light which will travel through the $2.5 \mu \mathrm{m}$ waveguide to the end. The guided light is collected from the cross section at the end of the waveguides. Figure 3 (c) shows the coupling and waveguiding effects of this structure. ${ }^{2}$

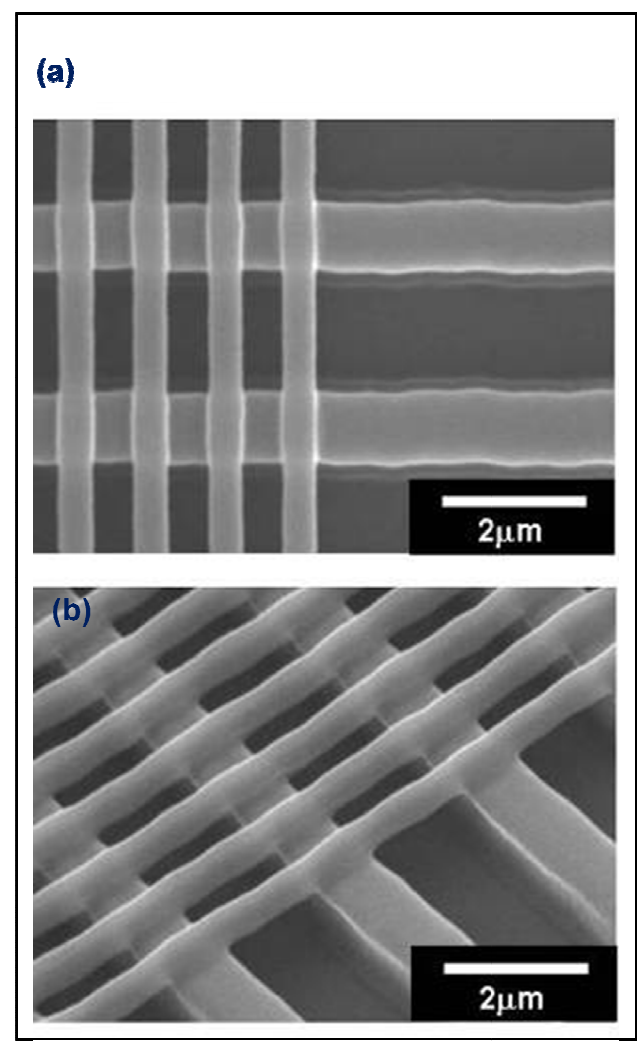

(c)

Coupling region Laser

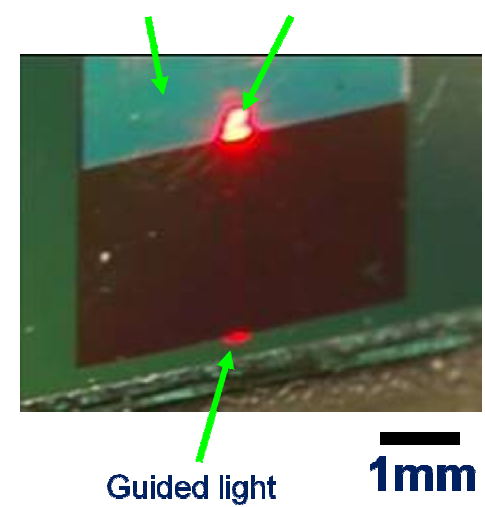

Figure 3 SEM images of (a) top and (b) diagonal views of grating coupler. (c) Laser illuminating the coupler region and guided by the waveguide. ${ }^{2}$ 


\subsection{Continuation from the previous work}

The same fabrication technique, $2 \mathrm{P}-\mu \mathrm{TM}$, is used as it provides an economical and well-developed method. The procedures of the fabrication will be altered slightly to fit the conditions and materials used. Instead of using the embedded quantum dots as the light source, external light sources and a coupler to couple the light into the waveguides will be used. In addition to a laser source, a white light source will also be used for the characterization of the waveguide. The white light provides a range of spectrum in the visible light which is more commonly available and usable. The ability of light by the waveguide to couple in different wavelengths can also be characterized using a white light source. 


\section{CHAPTER 3 - METHODS AND PROCEDURES}

This chapter introduces the basic principles of diffraction grating theory and the fabrication of the waveguide structure. The general fabrication method is introduced, and the procedures for the fabrication of the waveguide structure are explained in detail.

\subsection{Diffraction grating}

\subsubsection{Diffraction grating theory}

Diffraction of waves occurs when the waves encounter an obstacle with an opening comparable to the wavelength of the waves. In the case of light waves, including visible and infrared lights, with wavelength ranging from 400nm to 5000nm, diffraction occurs when the opening of the obstacle is in this range. A diffraction grating consists of an array of reflected or transmitted elements which are separated by a distance in the range of the wavelengths of light. ${ }^{6}$

There are two types of gratings, reflection gratings and transmission gratings. ${ }^{7}$ A reflection grating is used in this thesis for waveguiding purposes. Consider a wavefront encountering a grating structure separated by a spacing, d, as illustrated in Figure 4, with two light rays coming from the left side of the diagram incident on the grating structure. These two waves are in phase at wavefront A and are diffracted by the grating. By the principle of constructive interference, if the sum of the path lengths, $d \sin \theta_{1}$ and $d \sin \theta_{2}$, of the two waves is an integral number of the wavelengths, these two waves are in phase at wavefront $\mathrm{B}$. This results in the grating equation, $d \sin \theta_{1}+d \sin \theta_{2}=m \lambda$, where $\mathrm{m}$ is the diffraction order, which is an integer and can be positive, negative, or zero. The 
zeroth order simply means that it is a reflection. ${ }^{7}$ The sign convention used here is that it is positive on the side of the incident waves and negative on the opposite side of the normal where waves are diffracted.

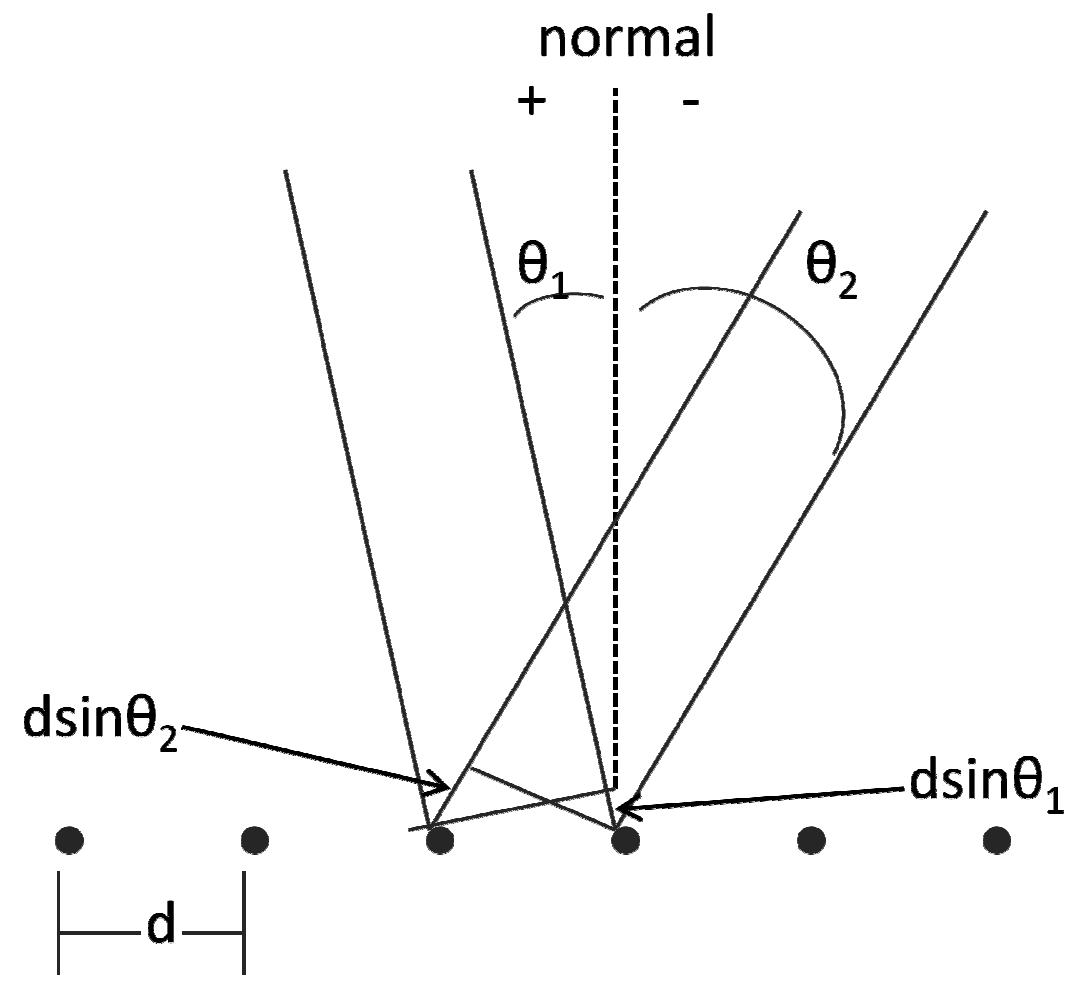

Figure 4 Illustration of the theory of diffraction for a planar wavefront.

The waveguide structure and proposed work in this thesis deal mainly with the diffracted light at the opposite side of the normal from the incident light. Therefore, the diffracted angle, $\theta_{2}$, and $\mathrm{m}$ are both negative while performing calculation. 


\subsection{Fabrication}

The fabrication technique used to make the coupler waveguide is called twopolymer microtransfer molding method. This technique was first developed for fabricating the photonic band gap materials with multi-layers. ${ }^{8}$

\subsubsection{Two-polymer microtransfer molding}

Polymer waveguides requires a highly ordered structure. There are a number of very well developed techniques to make complex structures at the micron and submicron scale, most of which were developed and are currently used by the semiconductor fabrication industry. However, most are very time consuming, require very specialized and costly equipment, and require that a clean room environment be used. Because of these restrictions, a new technique called two-polymer microtransfer molding $(2 \mathrm{P}-\mu \mathrm{TM})$ method is used to fabricate the polymer waveguides. ${ }^{8}$ This method was previously developed at Iowa State University (ISU) for the purpose of constructing photonic band gap materials. The advantages of this method are the low cost of the materials and equipment setup, ability to fabricate structure in open air, flexibility in design, and a broad selection and compatibility of the polymers. ${ }^{8}$

In $2 \mathrm{P}-\mu \mathrm{TM}$, two $\mathrm{UV}$ curable prepolymers are used, polyurethane (PU) and polymethacrylate (PA). PU is the filler and forms the waveguide structure itself, and PA is the adhesive agent between PU and the substrate. A polydimethylsiloxane (PDMS) mold is first made using a silicon wafer master with a photoresist relief pattern on the wafer. Alternatively, the mold can also be made using a two-beam holography

technique. ${ }^{9}$ The PDMS mold is set up under an optical microscope for the filling process. A drop of PU prepolymer is placed on the PDMS mold near the pattern area. A fixed 
metal blade is set to be in contact with the prepolymer, but not touching the PDMS mold. The droplet of PU is dragged across the pattern area with a constant speed. Liquid PU prepolymer fills the channels of the pattern area on the PDMS mold. This procedure is referred to as wet-and-drag (WAD). The blade serves to move the PU droplet to fill the channels on the mold instead of scraping the prepolymer on the pattern area. Because the PDMS mold has very fine features, if the blade were to contact the mold, it would cause defects and destroy the pattern. To avoid this, the polymers are chosen such that the prepolymer wets the PDMS to such an extent that the channels are filled, but the top surfaces are not coated. So, no scraping to remove excess material is necessary.

After the first WAD procedure, the sample is cured using high intensity UV to partially solidify PU. The PA prepolymer is then coated on PU using a second WAD process. A silicon wafer substrate is placed on the mold, and the entire sample is exposed to low intensity UV for curing. PA acts as a bonding agent between the PU and the substrate. When peeling off the PDMS mold, the PU rods adhere to the substrate. Multilayer structures can be achieved by repeating this procedure and rotation the next pattern $90^{\circ}$ to the previous one. Figure 5 is a schematic diagram of the $2 \mathrm{P}-\mu \mathrm{TM}$ fabrication technique. Prepolymer A and Prepolymer B represent PU and PA respectively. ${ }^{8}$ 


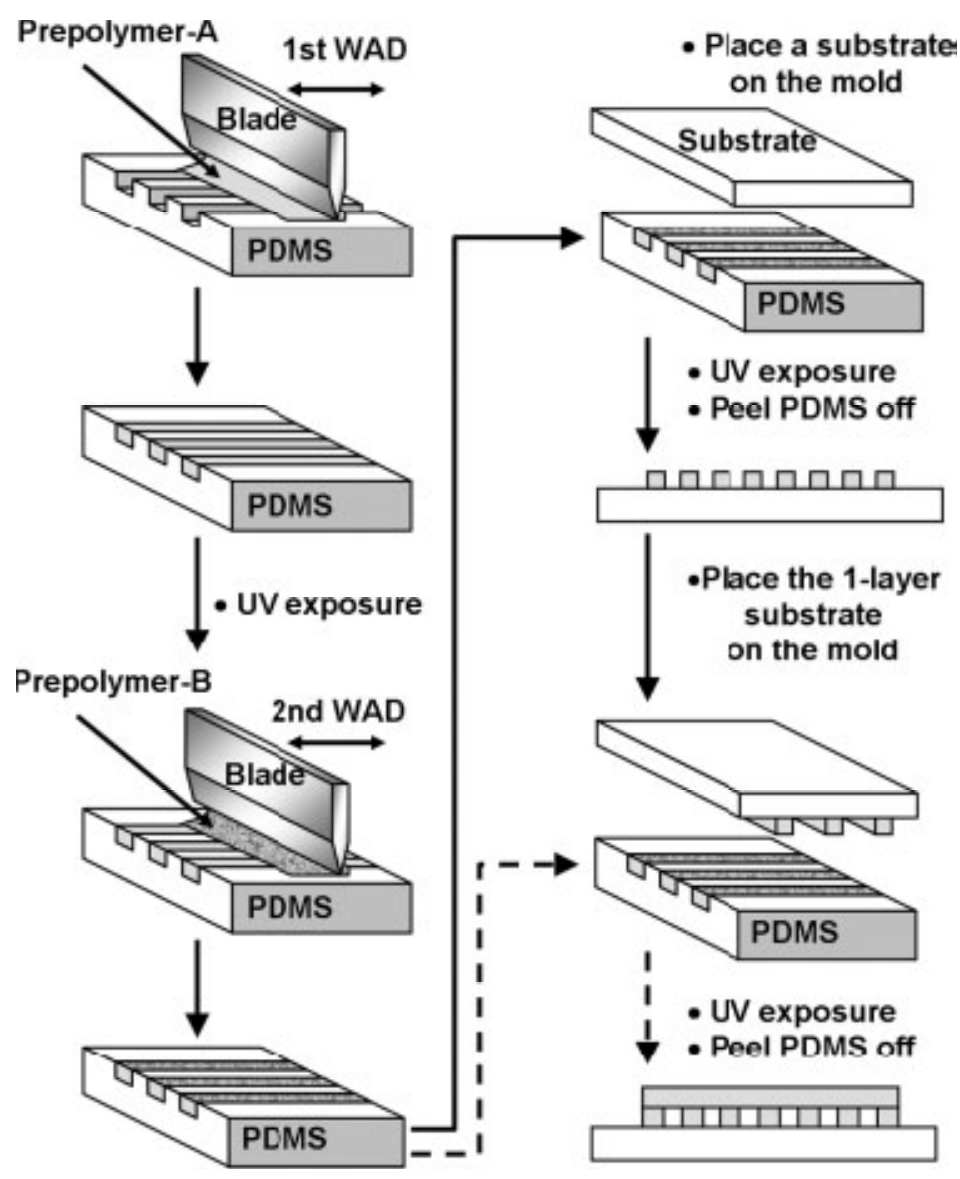

Figure 5 Two polymer microtransfer molding fabrication process. ${ }^{8}$ 
The entire procedure is done under an inverted optical microscope setup as shown in Figure 6.

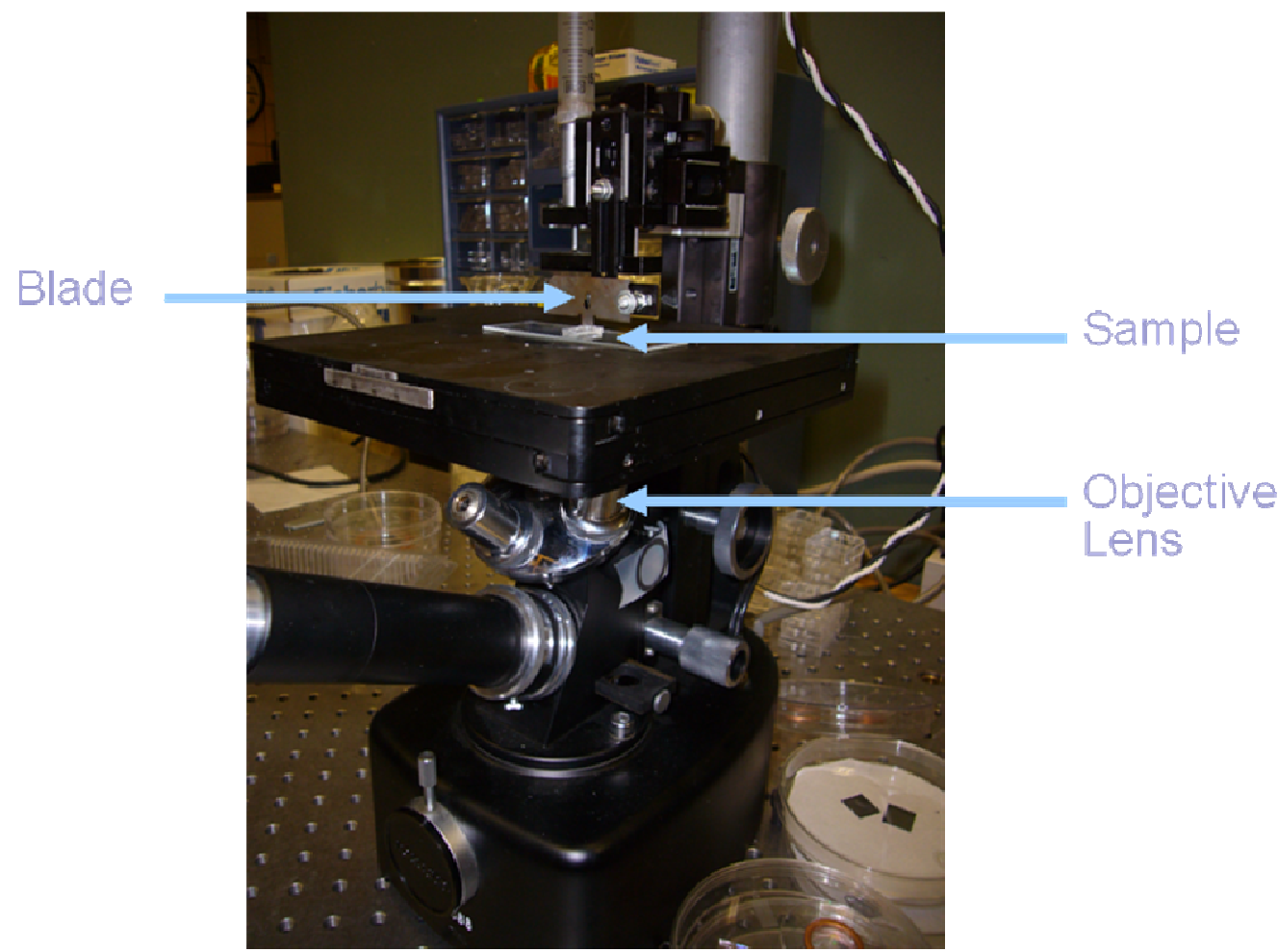

Figure 6 Experimental setup for 2P- $\mu \mathrm{TM}$.

The PDMS mold, PU, and PA are selected specifically for this process.

Polyurethane is selected as the prepolymer A due to its filling characteristics. The viscosity of PU is around 300cps, and the surface energy difference between PU and PDMS is large to allow even filling in the channels during WAD. ${ }^{8}$ When the PU drop is dragged across the pattern area, dewetting occurs on the PDMS surface between the channels. The PU in the channels will not be dragged along with the droplet because the adhesive energy of PU and patterned PDMS is larger than the cohesive energy of the PU. Polymethacrylate is chosen to be the bonding prepolymer B for several reasons. PA 
exhibits a selective wetting property to PU but not to PDMS. Combined with the low viscosity of PA, it forms only a thin layer coating on PU after WAD. Additionally, PA provides sufficient adhesive strength between PU and the substrate to allow the PU rods stay on the substrate while peeling off the mold.

\subsubsection{Coupler waveguide by two-polymer microtransfer molding}

The fabrication procedures are altered slightly for the waveguide structure. The structure consists of a $2.5 \mu \mathrm{m}$ PU grating on the silicon wafer with $2 \mu \mathrm{m}$ thick silicon oxide layer and another $1 \mu \mathrm{m}$ grating perpendicularly oriented on top of the $2.5 \mu \mathrm{m}$ waveguide.

During the first WAD PU filling, the $2.5 \mu \mathrm{m}$ PDMS mold is only filled about onesixth from one end and is put into high intensity UV chamber for pre-curing. The precuring only takes 15 seconds to partially solidify PU. This gives some rigidity to the PU so that during the second WAD, the PU will stay in place at this end of the mold. After pre-curing, a second WAD is done throughout the entire pattern area from the other end. A four-minute curing time is required to fully solidify PU for the $2.5 \mu \mathrm{m}$ pattern. It is found that the regular PA bonding agent between the pattern and the wafer substrate does not have enough adhesive power and results in poor yield. A ratio of 3:7 PU:PA mixture is used as the bonding agent where the function of PU is to increase the adhesive strength. After the WAD of the mixture, the mold is put on the substrate and sent in to the low intensity UV for a three-hour curing.

A second layer of $1 \mu \mathrm{m}$ periodicity pattern is made on top of the $2.5 \mu \mathrm{m}$ pattern.

The $1 \mu \mathrm{m}$ grating pattern is turned perpendicular to the direction of the $2.5 \mu \mathrm{m}$ pattern, and 
a part of it overlaps the $2.5 \mu \mathrm{m}$ pattern as shown in Figure 7. This overlapping region is the light coupler.

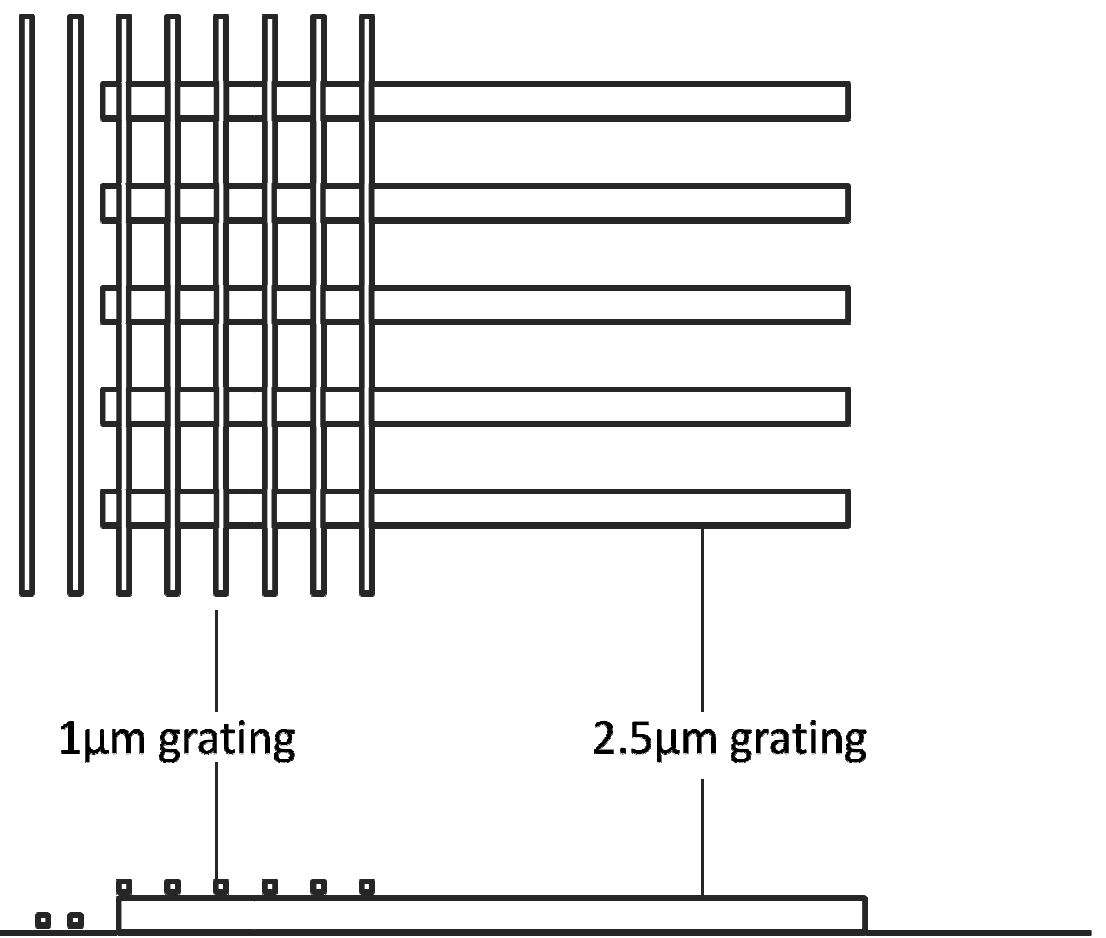

silicon oxide wafer substrate

Figure 7 Schematic diagram of the coupler waveguide structure.

The $1 \mu \mathrm{m}$ pattern does not require the first WAD as the $2.5 \mu \mathrm{m}$ pattern because of the smaller filling channels. The high intensity UV curing after the PU WAD is reduced to two and half minutes. The same bonding agent (PU:PA=3:7) is used.

\subsection{Characterization}

\subsubsection{Method}

In accordance with the diffraction theory in section "3.1 Diffraction Grating", an incoming light source will first be diffracted by the $1 \mu \mathrm{m}$ top layer. When the diffraction angle is at $90^{\circ}$, the diffracted light at the negative side of the normal will travel through 
the $2.5 \mu \mathrm{m}$ PU channels to the other end of the waveguide. ${ }^{6}$ According to the diffraction equation, , the second term is the diffracted light which equals to 1 when $\theta_{2}=90^{\circ}$. Since diffraction occurs at the other side of the normal, it is negative. The periodicity of the grating, $\mathrm{d}$, is $1 \mu \mathrm{m}$. Therefore, this suggests that for a certain incident angle, a specific wavelength of light will be diffracted in the direction of the waveguide. Figure 8 shows a schematic diagram of this concept and how the waveguide structure works. The light is first diffracted by the $1 \mu \mathrm{m}$ pattern, and the diffracted light travels inside the PU bars of $2.5 \mu \mathrm{m}$ pattern to the end of the waveguide.

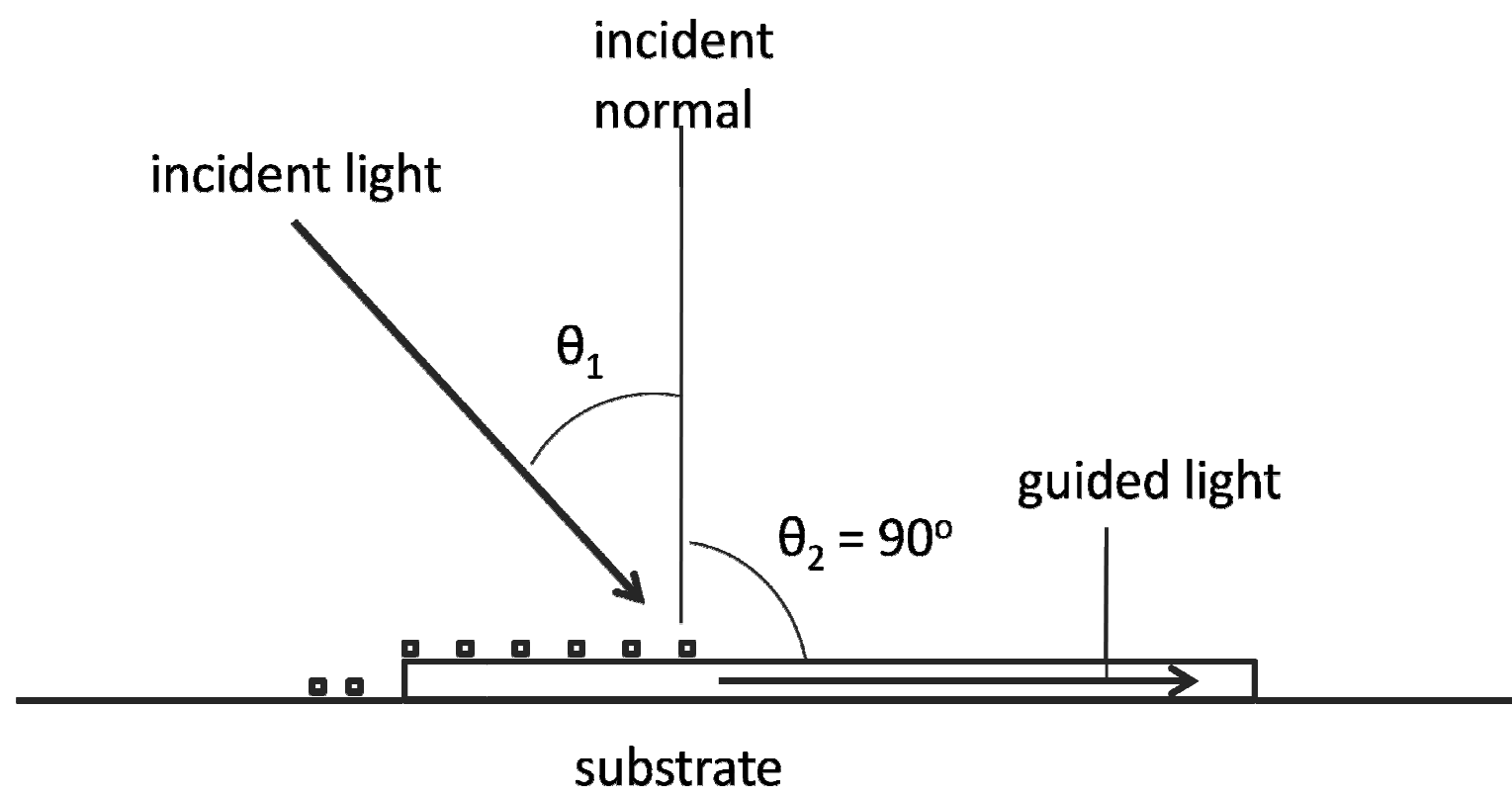

Figure 8 Conceptual representation of the function of the waveguide structure.

The light is guided and traveled through the PU bars of the $2.5 \mu \mathrm{m}$ pattern by total internal reflection. In order for total internal reflection to occur, the medium where the light is traveling through should be at a higher index of refraction than that of the surrounding medium. ${ }^{10}$ In addition, the index of refraction contrast needs to be high 
enough. The refractive index of the silicon wafer is much higher than that of the polyurethane; therefore, a film of silicon oxide layer is grown on top of the silicon to provide a lower refractive index to the PU bars. The index contrast, contrast $=$ $\left(n_{\text {core }}-n_{\text {cladding }}\right) / n_{\text {core }}$, is an important figure in optical waveguides. In the conventional fiber, the typical index contrast value is around $0.3 \% .{ }^{11}$ The index of refraction for silicon oxide is $1.46^{12}$ and $1.55^{13}$ for PU as from the manufacturer data. The index contrast, in this case, is about $6 \%$. This provides a high enough index contrast for the total internal reflection to occur. However, the silicon oxide layer has to be thick enough to prevent light penetration and loss. Hence, a $2 \mu \mathrm{m}$ thick silicon oxide layer is used as the substrate. The PU bars are separated by air which provides a high index contrast. No cladding is used between the PU bars.

\subsubsection{Experimental setup}

The experimental setup for the characterization of the coupler waveguide is shown in Figure 9. The pattern areas of both $1 \mu \mathrm{m}$ and $2.5 \mu \mathrm{m}$ are $4 X 4 \mathrm{~mm}^{2}$. A microscope is used to focus on the end of the waveguide where the light is guided through the PU channels. A charged-couple device (CCD) digital camera is mounted on the microscope to capture optical images of the guided light. In addition to the CCD camera, a spectrometer is mounted to characterize the spectra of the guided light. The model number of the spectrometer is USB2000 from Ocean Optics Inc. The usable range

of the spectrometer is $350-1000 \mathrm{~nm} .{ }^{14}$ 


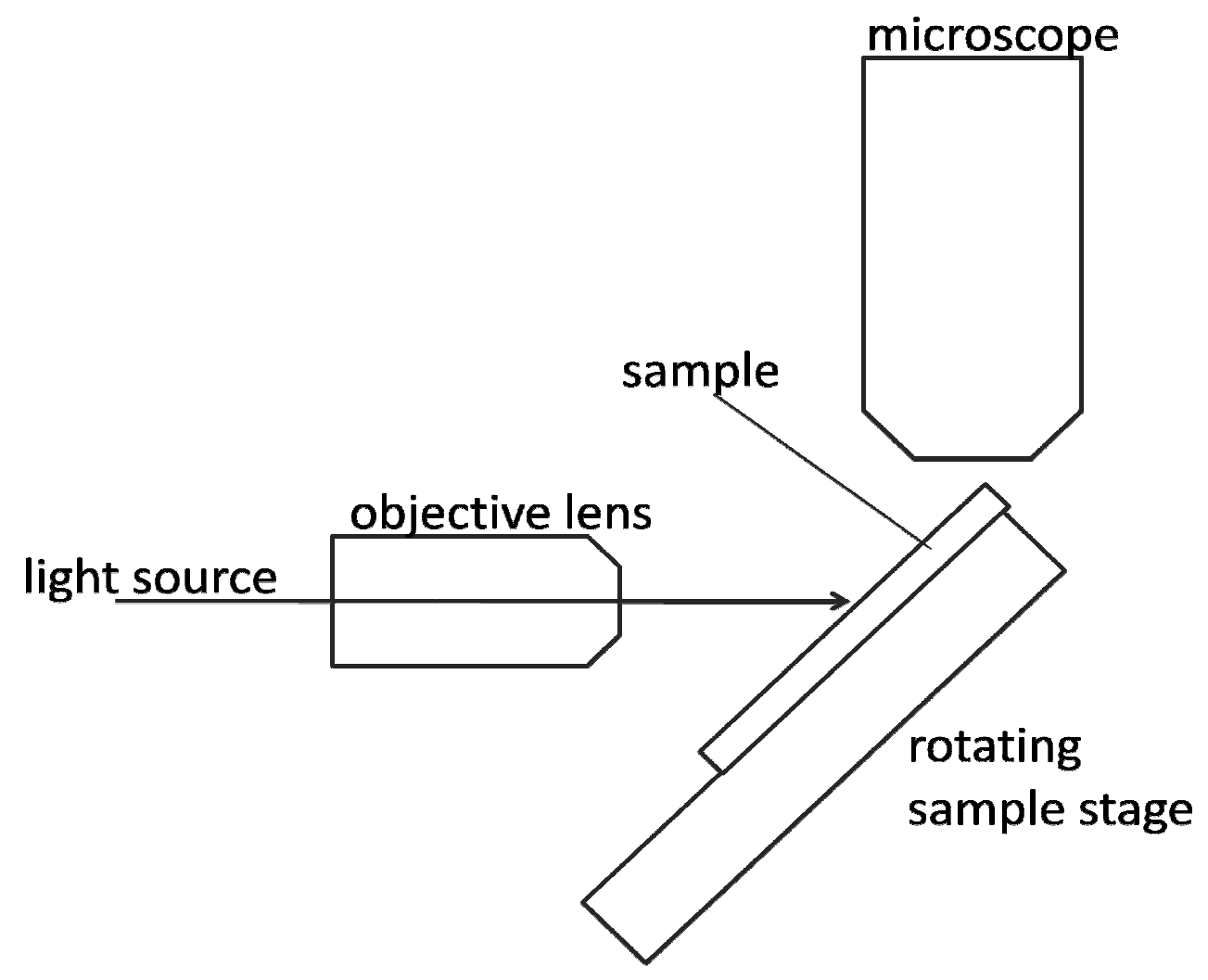

Figure 9 Schematic diagram of the experimental setup for characterizing the waveguide.

The light source is fixed in the horizontal direction. A rotating stage is used to alter the incident angle of the light. The light sources used are a helium lamp white light source and a He-Ne laser with wavelength equals $632.8 \mathrm{~nm}$. The laser beam impinges directly on the coupler region without the objective lens. When using the white light, an optical fiber is used to direct the light from the source onto the sample. The light exiting the optical fiber is dispersed; therefore, an objective lens is used to focus the light onto the coupler in order to achieve a small spot and a maximum intensity for better observation. The focused light spot is placed close to the edge of the coupler shown in Figure 10, which shows the front view of the waveguide sample. If the light spot is at the center of the coupler, when the light travels in the $2.5 \mu \mathrm{m}$ PU bars, the rest of the $1 \mu \mathrm{m}$ 
grating may act as another coupler to couple the light out. This might result in that no light is being guided through the waveguide.
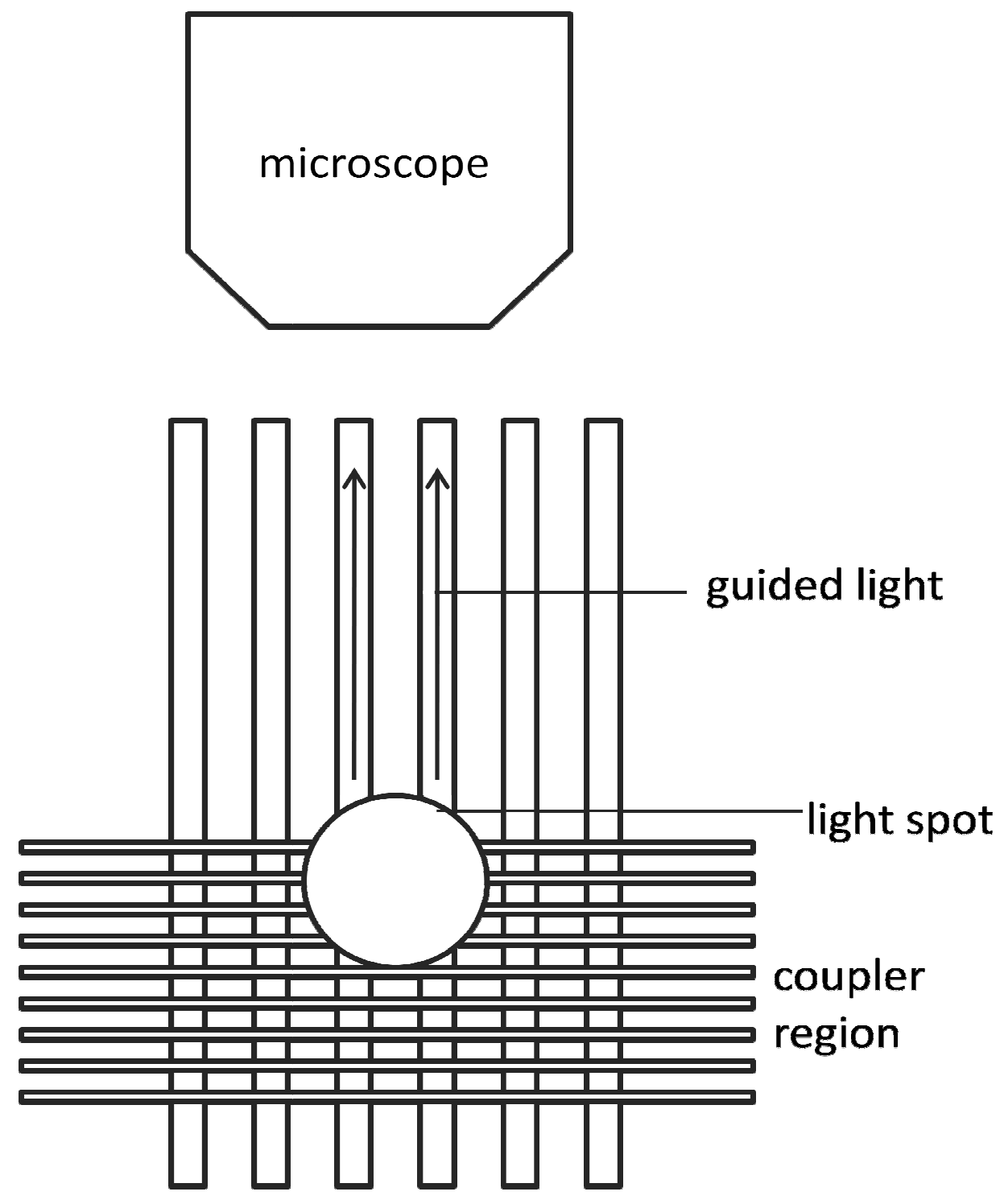

Figure 10 Schematic diagram showing the placement of the focused light spot on the coupler waveguide. 


\section{CHAPTER 4 - RESULTS}

The theoretical calculations are first done to predict the diffraction behavior of the waveguide structure. Then the experiments are performed to characterize the waveguides using both a laser and a white light source.

\subsection{Waveguide Structures Scanning Electron Microscopy}

Scanning electron microscopy (SEM) was done to show the structure and the dimensions of the waveguides. The waveguides are fabricated on a silicon wafer substrate with a silicon oxide layer. The area of the waveguide pattern is $4 \mathrm{X} 4 \mathrm{~mm}^{2}$. The SEM image of the cross section of the waveguide PU bars is shown in Figure 11.

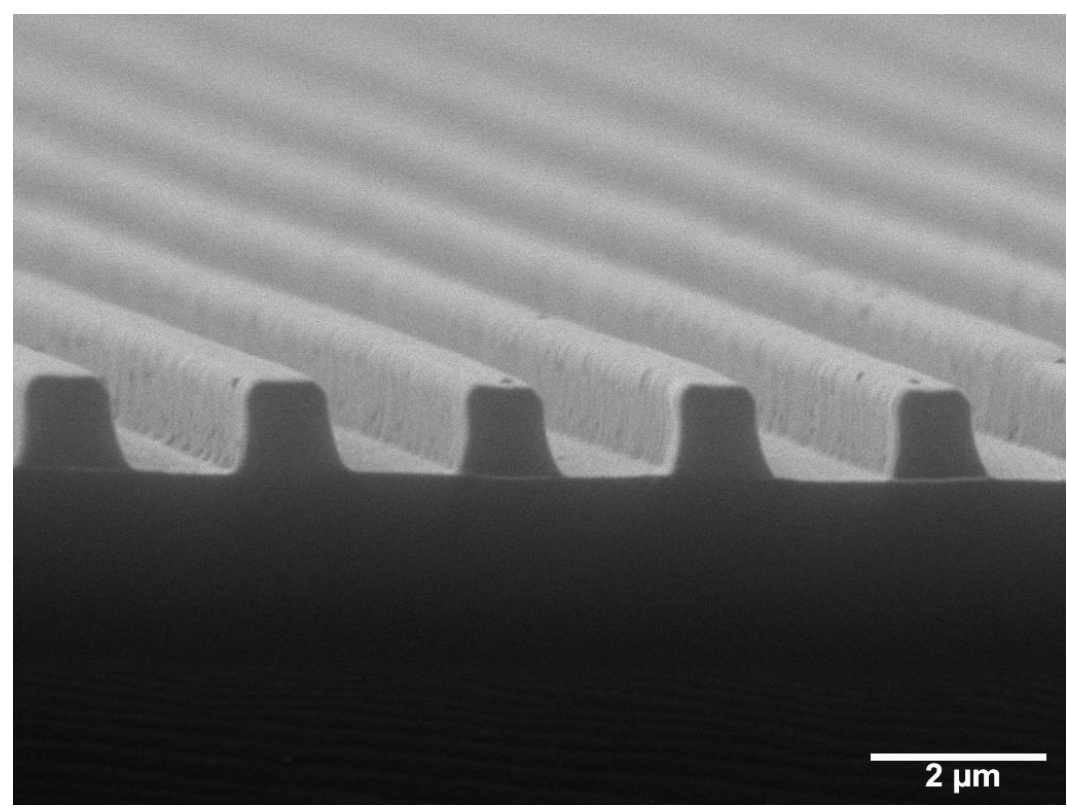

Figure 11 SEM image of the $2.5 \mu \mathrm{m}$ period waveguide structure. 
A $1 \mu \mathrm{m}$ periodicity pattern was fabricated perpendicular to and on top of the $2.5 \mu \mathrm{m}$ pattern as shown in Figure 12. This acts as the coupler to couple the external light source into the PU waveguides.

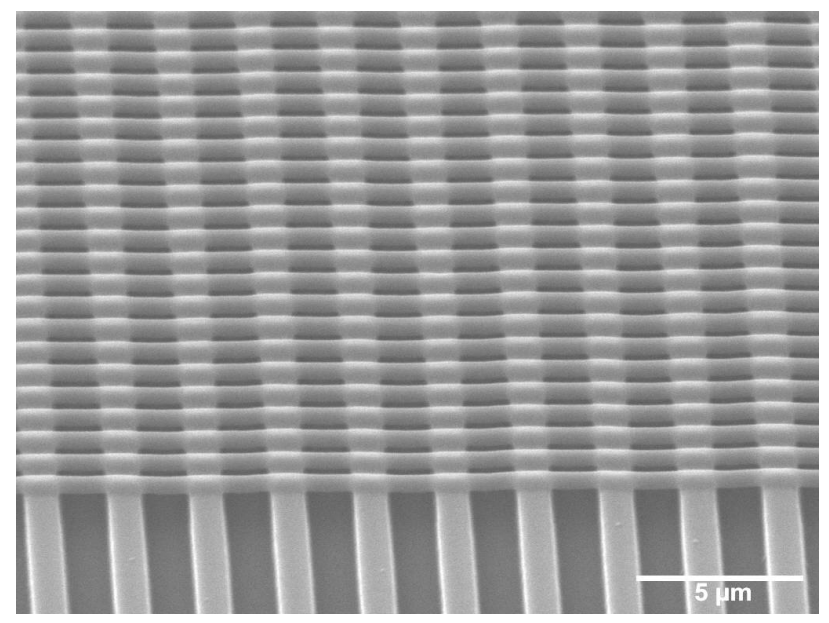

Figure 12 SEM image of the edge of the light coupler.

Figure 13 shows the cross section view of the $2.5 \mu \mathrm{m}$ period PU waveguides. The shape of the waveguides is trapezoidal. The width in the middle of the PU waveguides is about $1 \mu \mathrm{m}$, and the height is about $1.2 \mu \mathrm{m}$.

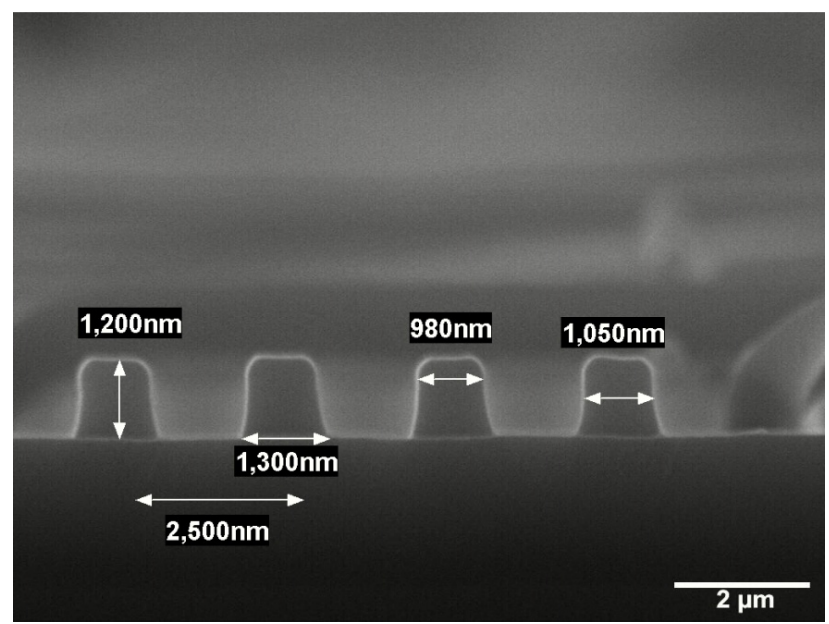

Figure 13 Cross section and dimensions of the waveguides. 


\subsection{Waveguide characterization using a laser}

\subsubsection{Experimental setup and theoretical calculations}

It is logical to first check the behavior of the coupler with a laser. The laser used is a helium-neon (He-Ne) laser with wavelength of $632.8 \mathrm{~nm}$. The laser beam is set to have a normal incident on the coupler region. The sample is oriented such that the $2.5 \mu \mathrm{m}$ PU bars are aligned vertically, and the $1 \mu \mathrm{m}$ bars are then in the horizontal direction. This is illustrated in Figure 14. A plain white paper is set in front of the sample to observe the diffraction pattern by the coupler. A small hole is made in the middle of the paper to allow a passage for the laser beam.

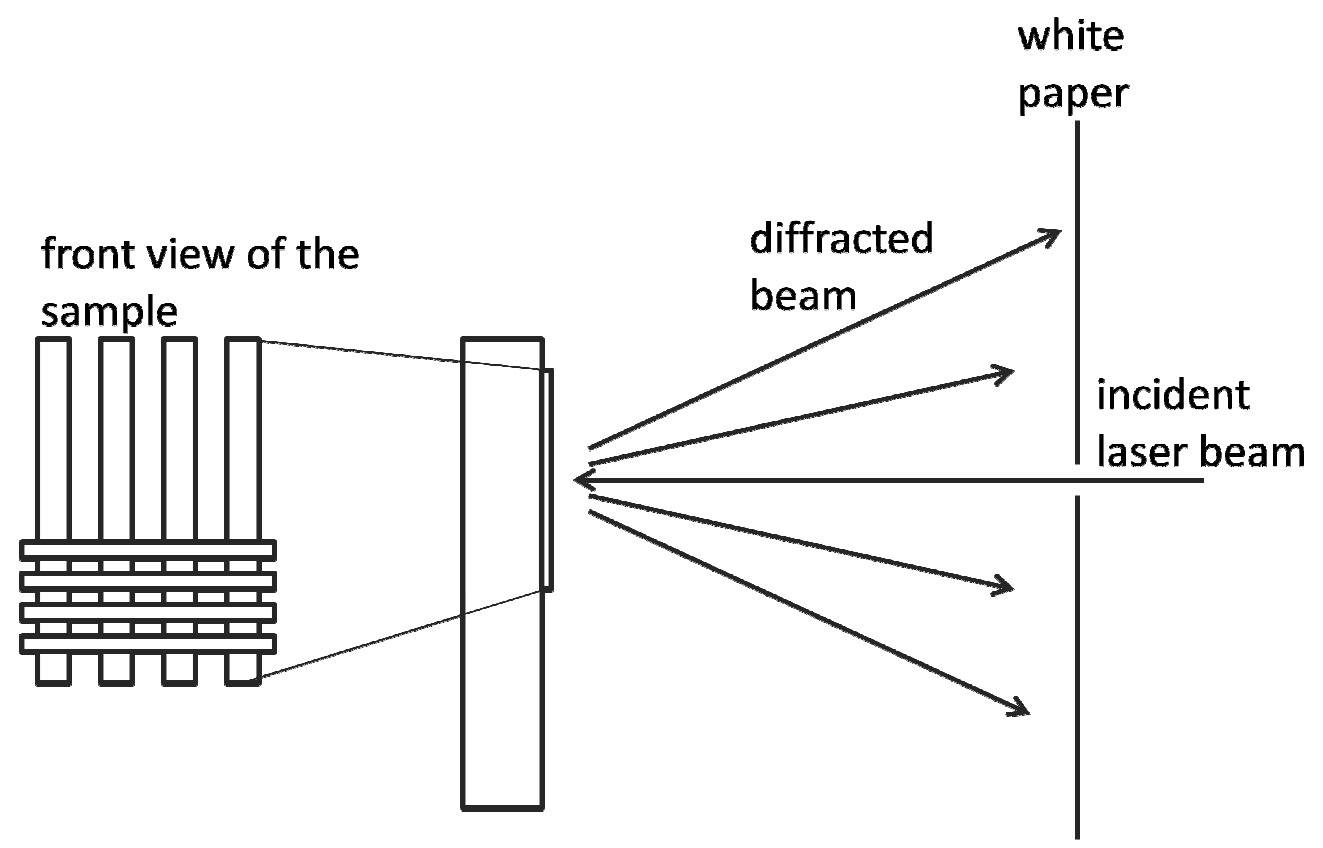

Figure 14 Experimental setup to observe the diffraction pattern with a normal incident laser beam. 
Due to the overlapping region of the two grating patterns and their perpendicular orientation, when a laser beam hits on this coupler, a two-dimensional diffraction pattern is created. The diffraction pattern along the vertical direction is from the $1 \mu \mathrm{m}$ grating, and horizontal pattern from $2.5 \mu \mathrm{m}$ grating. The diffraction from $2.5 \mu \mathrm{m}$ grating can be ignored because the diffracted light has not traveled along the direction of the $2.5 \mu \mathrm{m}$ PU bars.

The function of the $1 \mu \mathrm{m}$ grating is to diffract the light along the direction of the $2.5 \mu \mathrm{m}$ PU bars so that the light is guided. The first order diffraction has the highest intensity and easier to be observed after being guided. ${ }^{15}$ To calculate the diffraction angle of first order, the diffraction equation changes slightly due to the normal incidence. With the incident angle equals $90^{\circ}$, the diffraction equation becomes $d \sin \theta=m \lambda$. Rearranging the equation and plugging in $\mathrm{m}=1, \lambda=0.6328 \mu \mathrm{m}$, and $\mathrm{d}=1 \mu \mathrm{m}$, the angle of diffraction is calculated to be $39.3^{\circ}$.

$$
\begin{aligned}
& \sin \theta=\frac{m \lambda}{d}=\frac{(1)(0.6328 \mu m)}{1 \mu m} \\
& \theta=39.3^{\circ}
\end{aligned}
$$

According to the equation, the second or higher orders of diffraction do not exist as $\mathrm{m} \lambda>1$.

\subsubsection{Results using a laser beam}

Using the setup up shown in Figure 14, the diffraction pattern is seen on the white paper screen. This is demonstrated in the photographs in Figure 15. The horizontal diffraction pattern is due to the $2.5 \mu \mathrm{m}$ grating and the vertical from the $1 \mu \mathrm{m}$ grating. The second or higher orders of diffraction from the $1 \mu \mathrm{m}$ grating are not observed as is predicted 
by the diffraction equation.

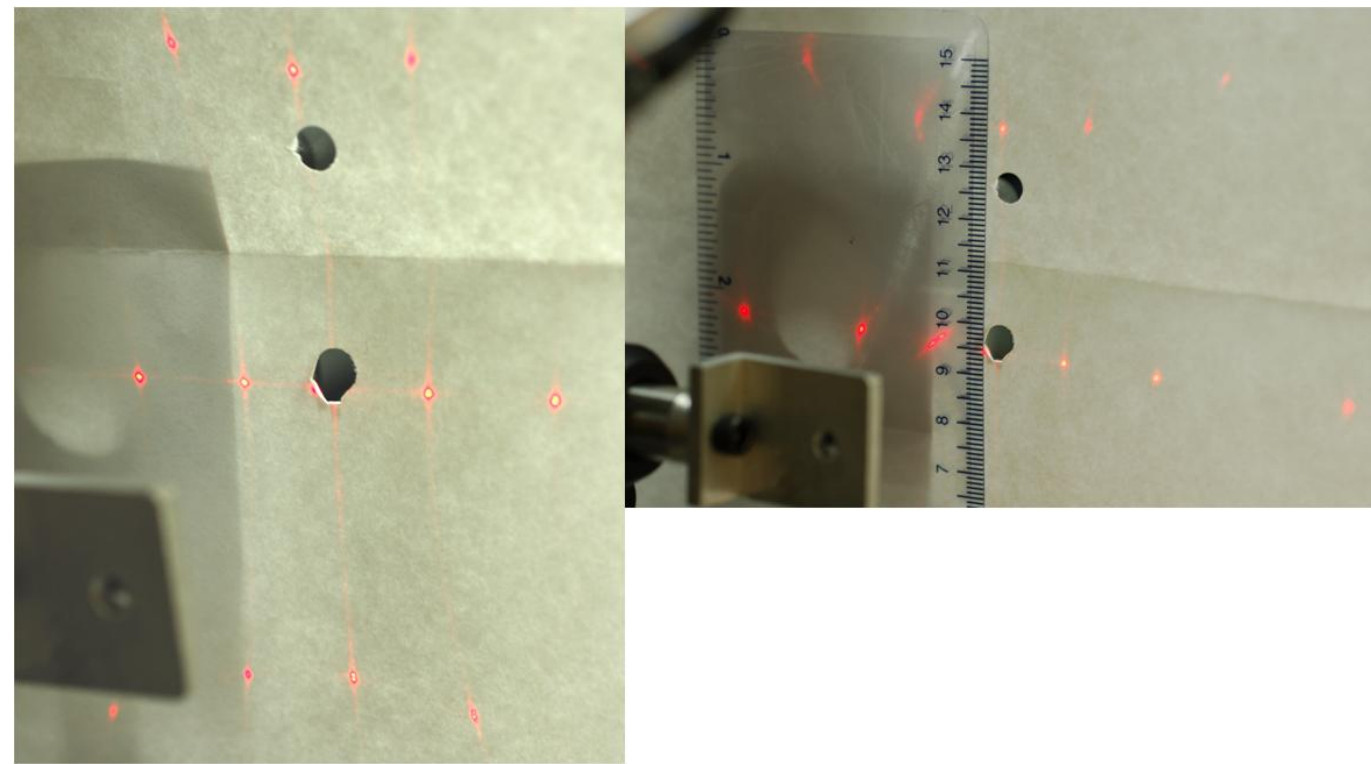

Figure 15 (a) The diffraction pattern from the coupler is imposed on the paper screen. (b) The distance from the incident beam to the 1st order diffraction is measured.

In order to find the first order diffraction angle, the lengths from the incident laser beam and the first order diffraction light as well as the distance from the sample plane to the paper screen are measured. These measured distances are expressed in centimeters and shown in Figure 16. 


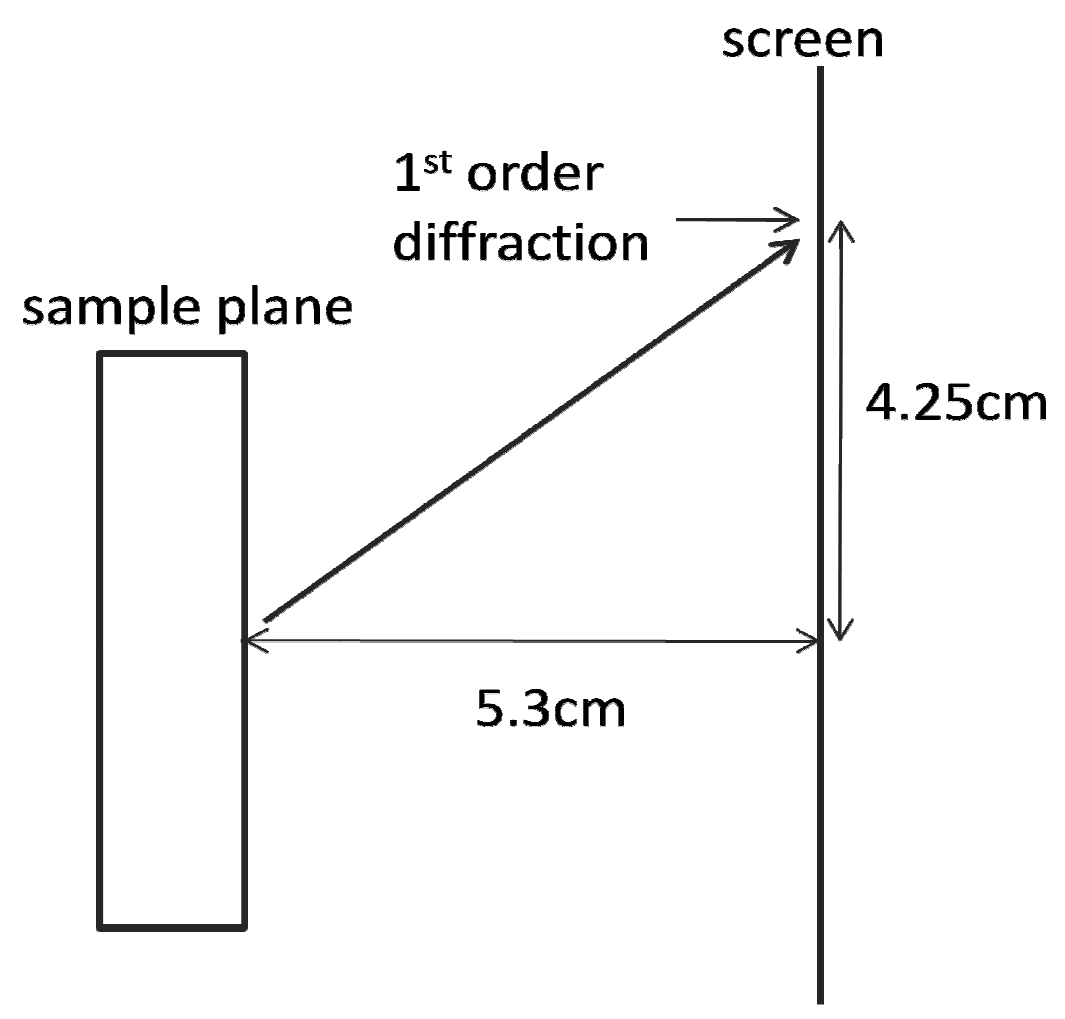

Figure 16 Measured distances to calculate diffraction angle.

Using the tangent function, the angle is calculated to be $38.7^{\circ}$.

These experimental results correspond to the theoretical calculation very well. The small difference between the theoretical and experimental angles may be due to that the incident laser beam slightly deviates from the horizontal position as well as the measurement errors. 
After the diffraction pattern is obtained for normal incidence, the sample is rotated so that the diffraction angle for the first order diffraction is at $90^{\circ}$. This results in the diffracted light traveling through the $2.5 \mu \mathrm{m}$ PU bars. The incident angle is turned to around $21.5^{\circ}$ which is the calculated angle. An optical image is taken using the setup described in Figure 9. Figure 17 is the picture taken showing that the first order diffracted light traveled through to the end of the waveguide.

Figure 17 Optical image of the guided 1st order diffraction at the end of the waveguide.

\subsection{Waveguide characterization using a white light source}

The light source was changed from a He-Ne laser to a white light helium lamp. This introduces the entire range of the optical visible spectrum and is used to characterize the ability of the waveguide to separate and guide a certain wavelength of light.

\subsubsection{Calculation and concepts}

\subsubsection{Guiding wavelengths}

The principal concept of the waveguiding capability of this structure is that, when the white light is incident on the coupler region, it is first diffracted by the $1 \mu \mathrm{m}$ pattern. The spectrum of the white light is then separated into different wavelengths, or colors, by the 
grating. At some incident angles, a specific wavelength will have a diffraction angle of $90^{\circ}$. This light will be coupled into and travel through the $2.5 \mu \mathrm{m}$ PU bars as illustrated in Figure 8 .

Due to the complexity of the two layer waveguide structure, the calculation is simplified to the fundamental principle of this waveguide structure. In order to identify the correct incident angle for a specific wavelength of light to be coupled, the diffraction grating equation, $d \sin \theta_{1}+d \sin \theta_{2}=m \lambda$, is used. In this case, a specific wavelength in microns, $\lambda$, is set, $\mathrm{d}=1 \mu \mathrm{m}$ because the diffraction from the $2.5 \mu \mathrm{m}$ pattern does not play a role in the guided light, $\theta_{2}$ equals $-90^{\circ}$ and $\mathrm{m}=-1$. The negative signs indicate that the diffracted light is on the opposite side of the normal from the incident light. First order diffraction is used because it provides the strongest intensity compared to higher order diffraction. Five wavelengths are chosen along the visible light range $(400 \mathrm{~nm}$ to $700 \mathrm{~nm}) .{ }^{16}$ The corresponding incident angles are calculated and summarized in Table 1. 
Table 1 The calculated incident angles for each of the five chosen wavelengths.

\begin{tabular}{|c|c|}
\hline wavelength $(\mathrm{nm})$ & calculated incident angle(degree) \\
\hline 450 & 33.4 \\
\hline 500 & 30.0 \\
\hline 550 & 26.7 \\
\hline 600 & 23.6 \\
\hline 650 & 20.5 \\
\hline
\end{tabular}

Due to the possibility of multi-order diffraction across the visible spectrum which may overlap with one of the chosen wavelengths, the incident angles are calculated for the first, second, and third order diffraction from 400nm to 700nm, and it is plotted in Figure 18. This shows that higher order diffractions do not overlap the first order and will not be observed for the chosen angles. This plot also shows that higher orders do not exist as the calculated incident angles are in the negative regime.

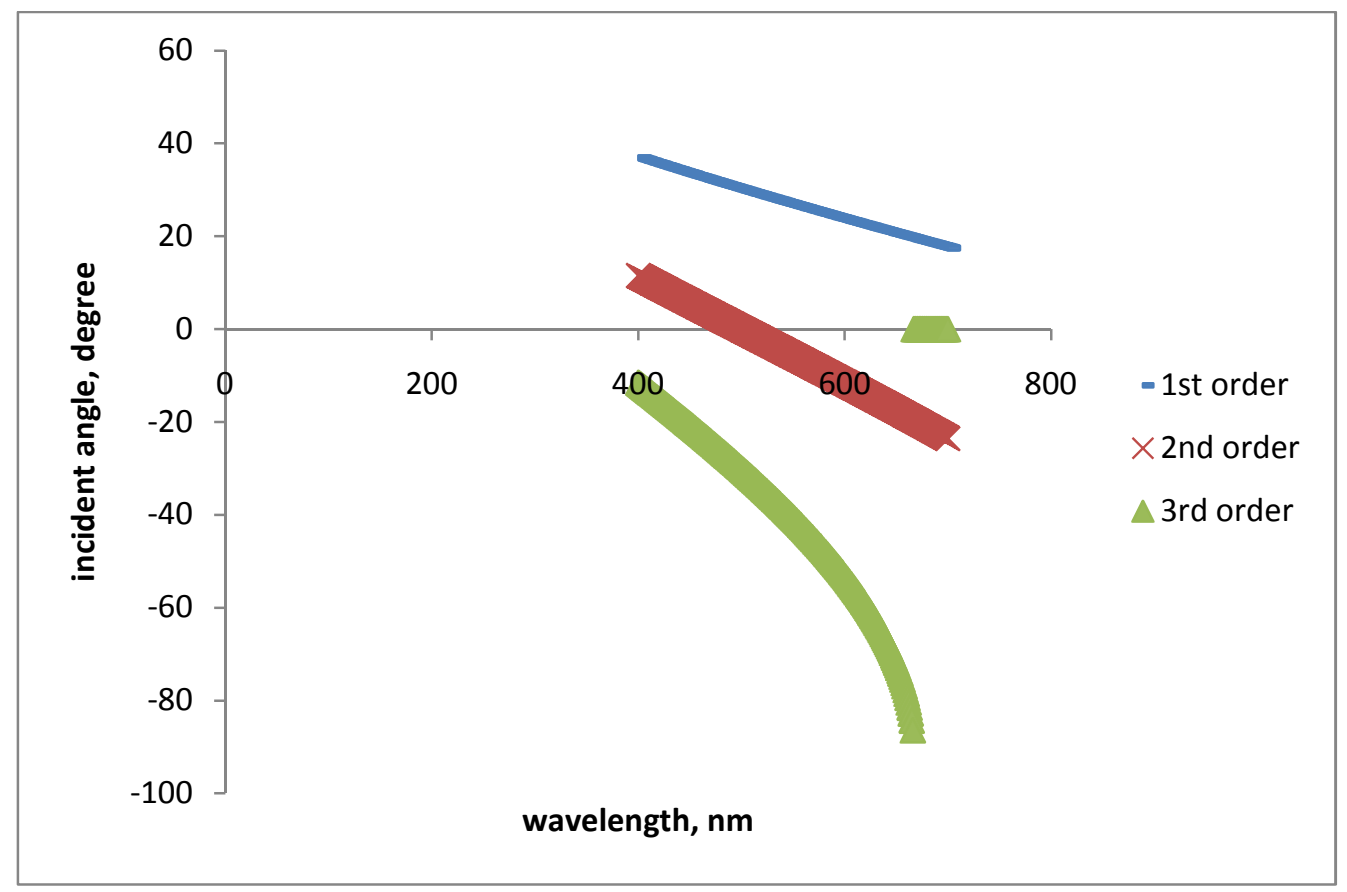

Figure 18 The calculated incident angles for multi-order diffraction in the visible range. 


\subsubsection{Peak broadening}

Using the white light source requires an objective lens to focus the light to provide a small spot and higher intensity as explained in section 3.3.2. The magnification of the objective lens used is 10X. It was experimentally determined that lower magnifications do not provide a small enough spot. For higher magnifications, the focal distance is too close to the sample which causes problems while rotating the sample. However, a problem arises from using an objective lens to focus the light. The incident rays of light are not parallel, but they become convergent. This results in a range of angles incident on the coupler and causes a broadening of the peak spectrum. This is illustrated in Figure 19.

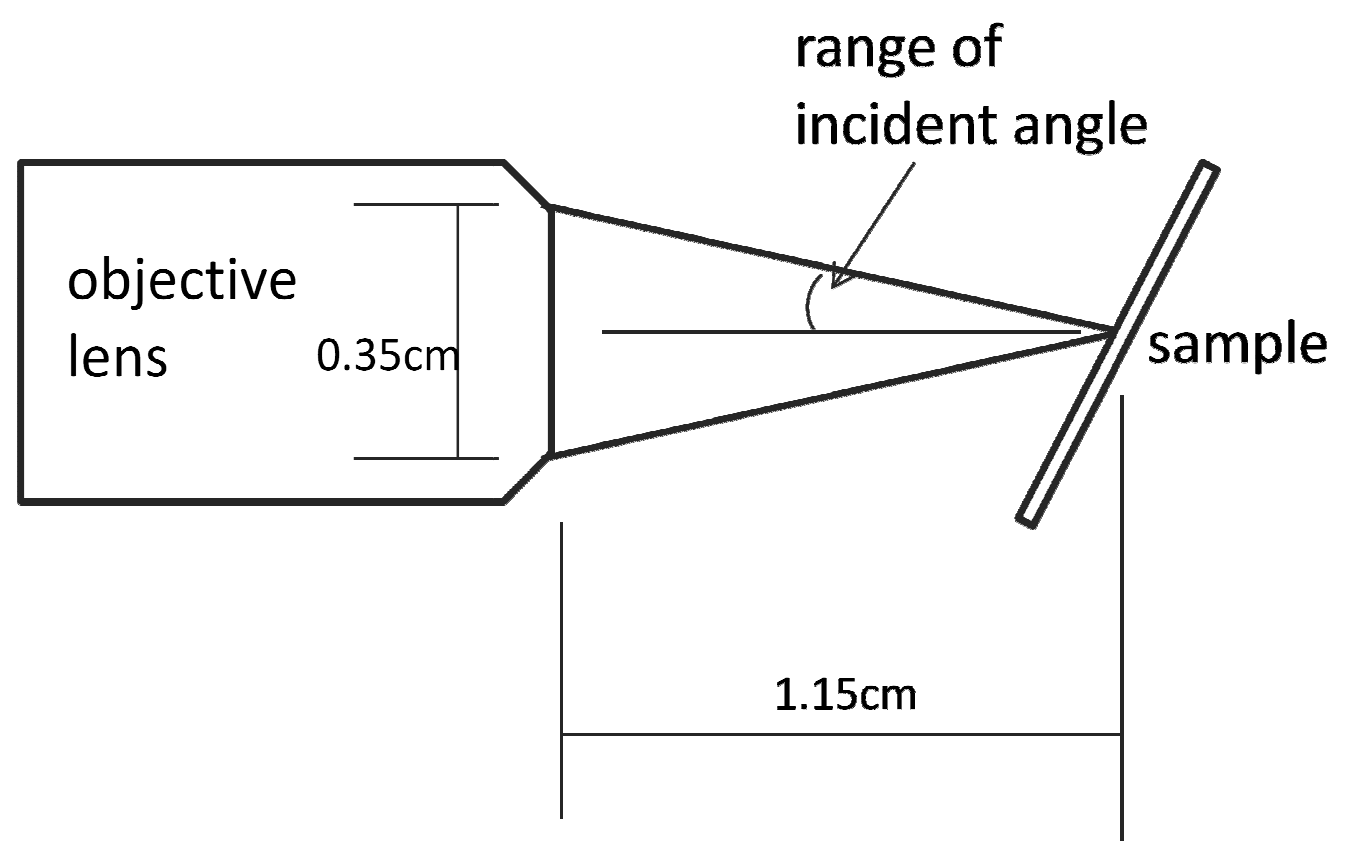

Figure 19 Broadening of peak spectrum due to convergent incident light.

This broadening of the peak can be estimated by knowing the range of the incident angles. The convergent angle can be calculated by measuring the distance from the sample to the objective lens and the length from the center of the objective lens to its end. The 
measurements are shown in Figure 19. The angle is then calculated as follows.

$$
\begin{aligned}
& \tan \theta=\frac{\text { opposite }}{\text { adjacent }}=\frac{0.350 / 2 \mathrm{~cm}}{1.15 \mathrm{~cm}} \\
& \theta=8.65^{\circ}
\end{aligned}
$$

Using the angle calculated above, the broadening of the peaks effect can then be calculated. An example calculation is as follows using the selected wavelength of 500nm.

$$
\begin{aligned}
& d \sin \theta_{1}+d \sin \theta_{2}=m \lambda \\
& (1 \mu m) \sin \left(30^{\circ}+8.65^{\circ}\right)+(1 \mu m) \sin \left(-90^{\circ}\right)=(-1) \lambda \\
& \lambda=0.3754 \mu m=375.4 n m \\
& (1 \mu m) \sin \left(30^{\circ}-8.65^{\circ}\right)+(1 \mu m) \sin \left(-90^{\circ}\right)=(-1) \lambda \\
& \lambda=635.9 \mathrm{~nm}
\end{aligned}
$$

This wavelength range is calculated for each of the selected wavelengths. These broadening effects are plotted in Figure 20. The x-axis represents the broadening ranges corresponding to the selected wavelength on the y-axis. The calculated incident angles are also shown with the equivalent selected wavelengths. 


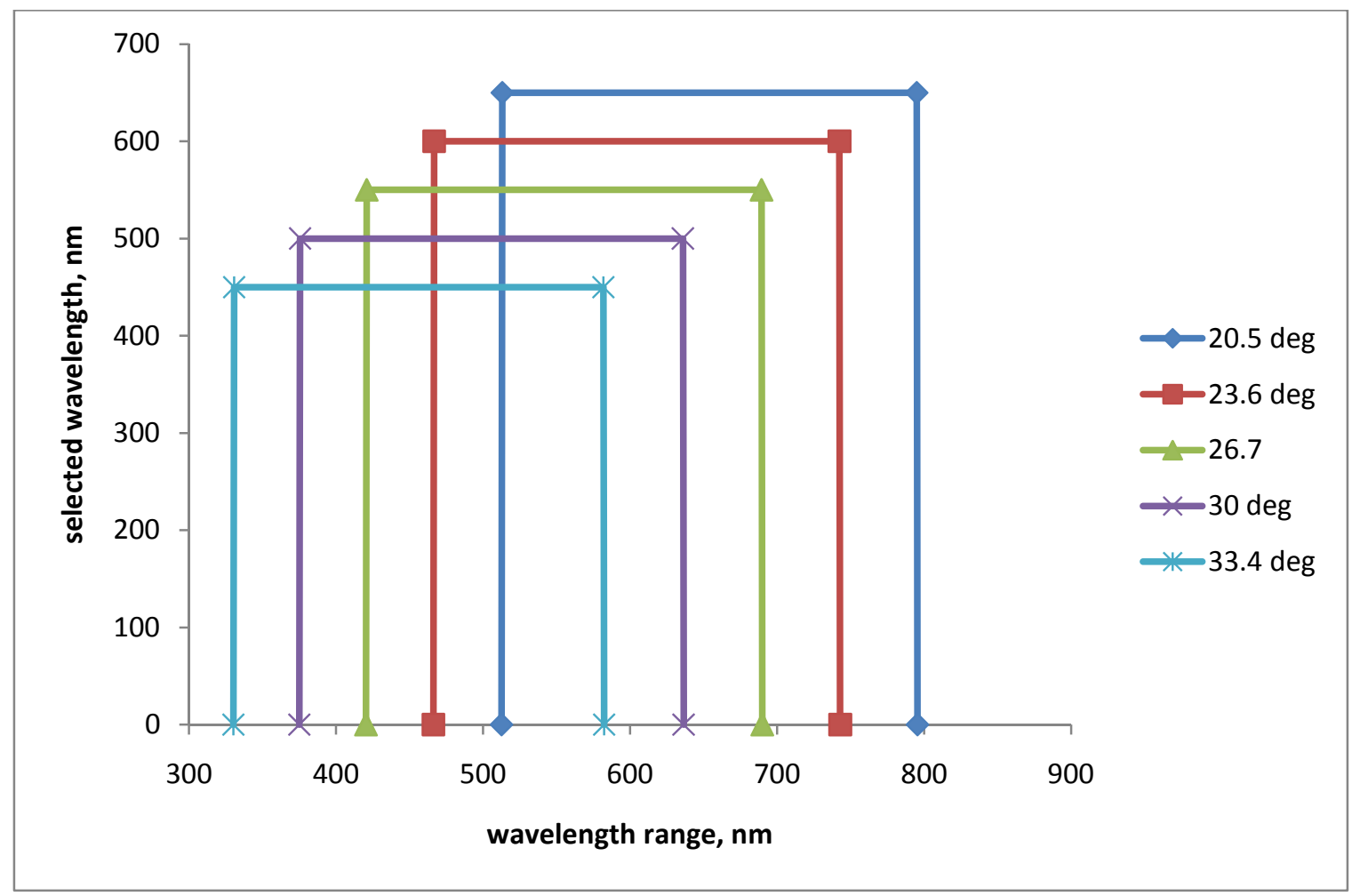

Figure 20 Convergent lens effect of peak broadening for each selected wavelength.

\subsubsection{Results using white light source}

\subsubsection{Source Spectrum}

The white light source used is a tungsten halogen lamp. The spectrum of the source was first determined. The spectrum of the light source has a range from around $350 \mathrm{~nm}$ to $1000 \mathrm{~nm}$ as shown in Figure 21. This source may not provide enough information in the higher frequency region because of the low intensity. 


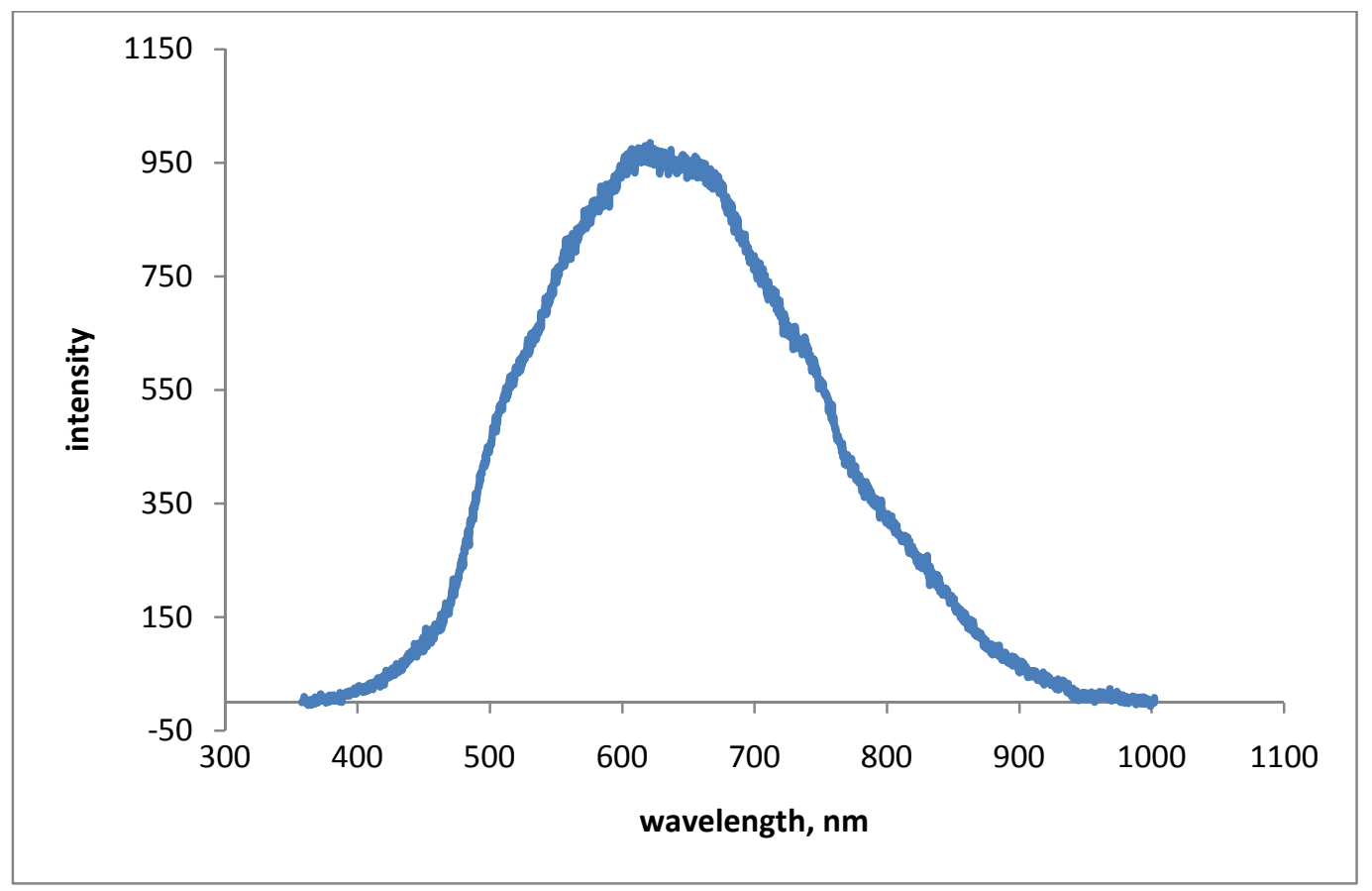

Figure 21 Spectrum of the halogen lamp light source.

\subsubsection{Focused light beam position}

After determining the source spectrum, the experiment using the calculated incident angles in Table 1 was performed. However, it was found that the location of the focused light spot on the coupler affects the spectrum greatly. The guided light at the end of the waveguide is only observed when the light spot is on or near the edge of the coupler. This phenomenon can be explained that if the light spot is not near the edge of the coupler, the proceeding $1 \mu \mathrm{m}$ PU bars can act as out-couplers which diffract the light out of the waveguide.

As a result, there is no guided light observed. If the light spot is above the coupler and incident on the $2.5 \mu \mathrm{m}$ pattern, there will be no diffraction by the $1 \mu \mathrm{m}$ pattern. A series of tests was done to investigate the behavior of the location of the light spot on the light guiding ability. The light spot is first placed just below the coupler edge. The spectrum and an optical image are taken. Then the light spot is moved higher where a part of the spot has 
crossed the coupler edge. The data are again collected. This is repeated until the light spot is above the coupler edge. Figure 22 is a schematic diagram showing the position change of the light spot.

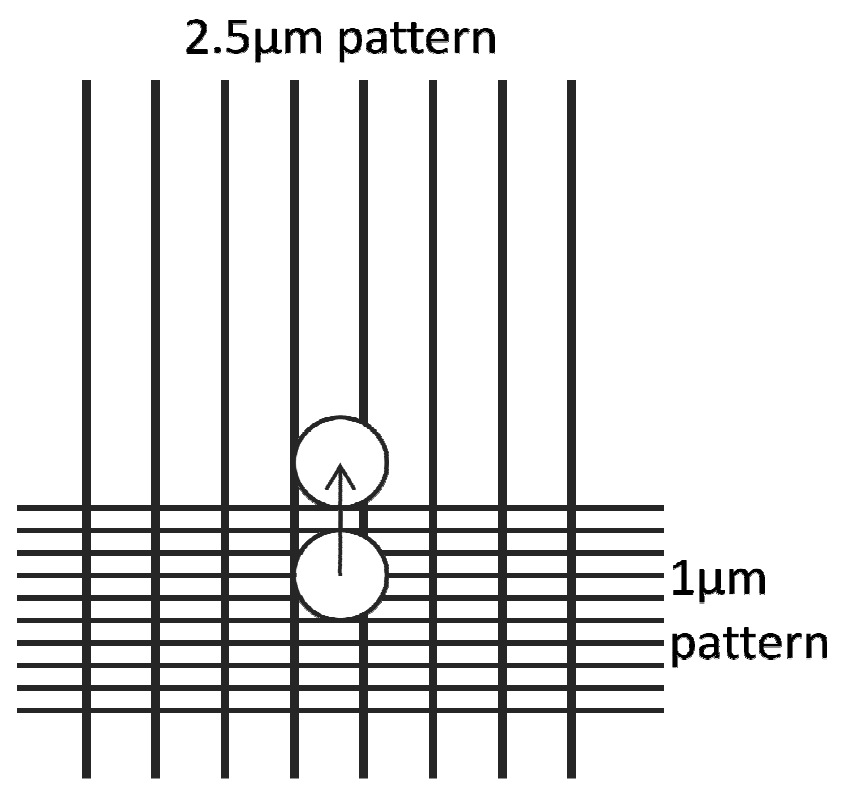

Figure 22 Movement of the light spot from within to out of the coupler.

The spectra and optical images are taken for four positions of the light spot. The incident angle used is $27^{\circ}$ which corresponds to a guided wavelength of 546nm. Figure 23 shows the optical images of the guided light at the end of the $2.5 \mu \mathrm{m}$ bars. The individual bars are seen as the brighter spots in the pictures. The first image is taken when the light spot is within the coupler. The color of the guided light changes from green to yellow to orange as the light spot moves out of the coupler. 


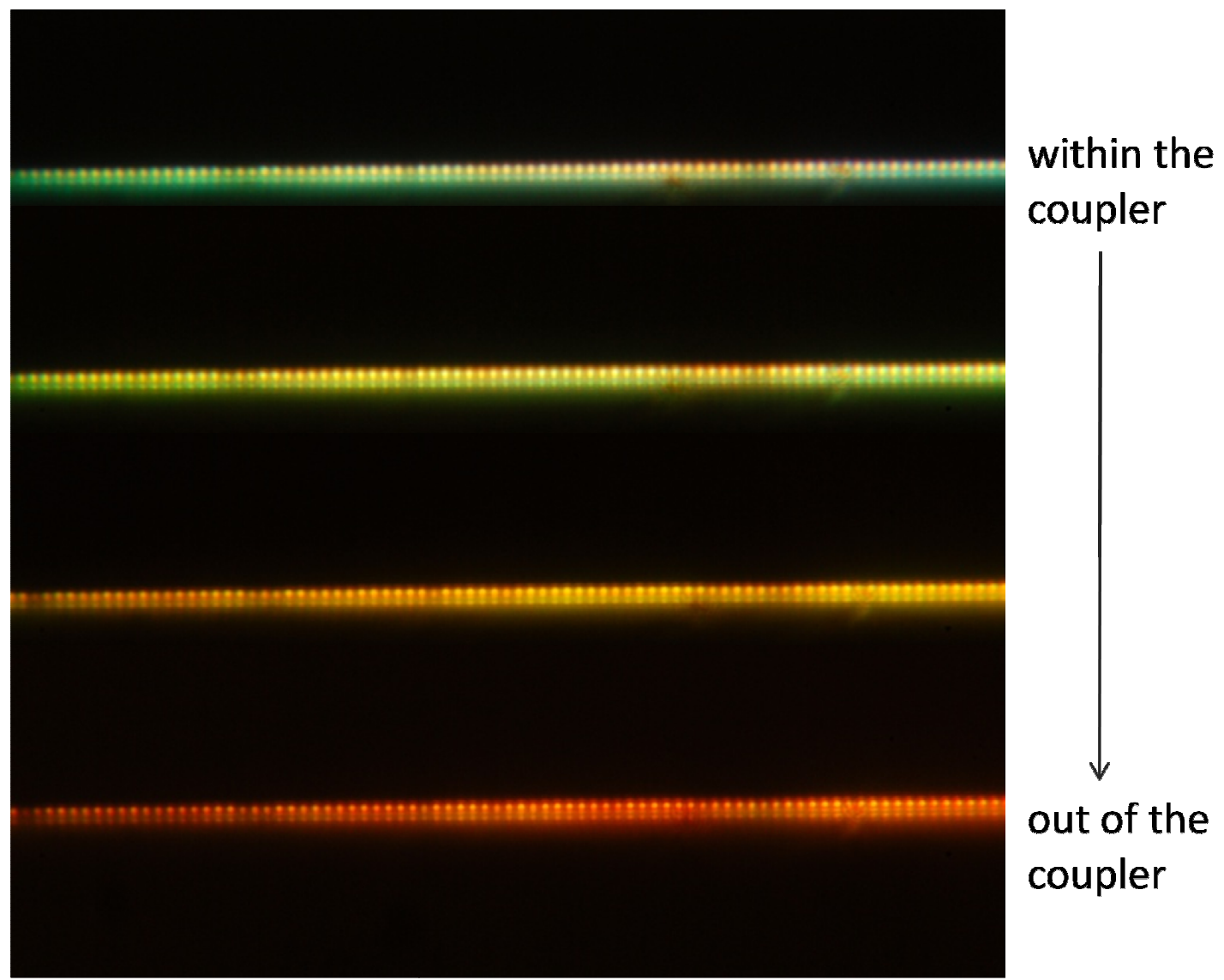

Figure 23 Optical images of the guided light with varying light spot position.

The spectra are also taken following this progression and are shown in Figure 24. The spectrum of "rising 1" indicates that part of the light spot has crossed the coupler edge onto the $2.5 \mu \mathrm{m}$ pattern region, and "rising 2 " means that most of the light spot is on the $2.55 \mu \mathrm{m}$ pattern. 


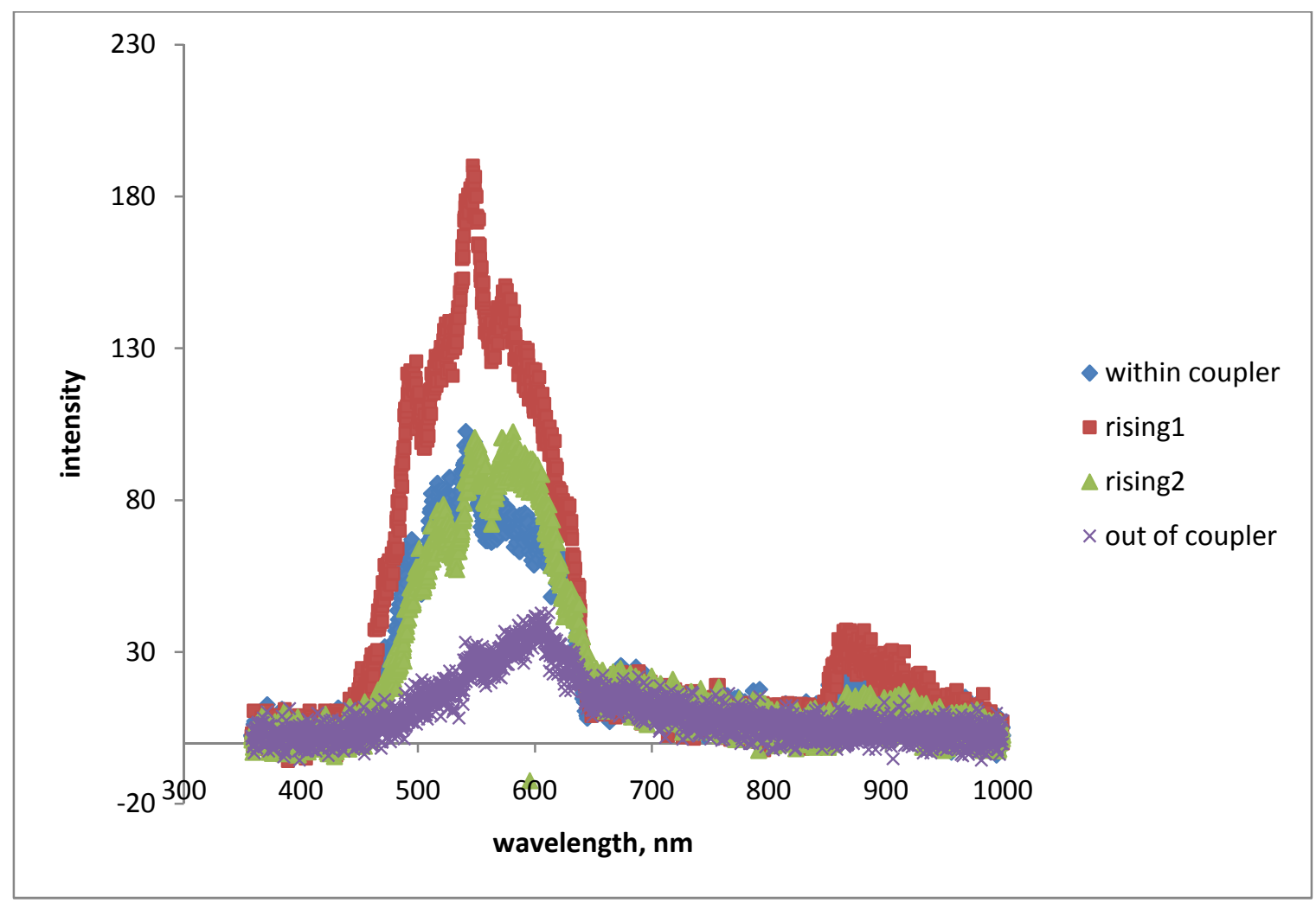

Figure 24 Spectra of the different position of the light spot on the coupler.

It is clear from the spectra that when the spot or part of the spot is on the coupler region, the calculated wavelength of $546 \mathrm{~nm}$ is coupled in and travels through the waveguide. This corresponds to the optical images as the green color is observed. The maximum intensity is only observed when the light spot is on the coupler edge as shown in "rising 1". However, when the spot moves out of the coupler, there is no coupling observed of the calculated wavelength. It is further determined that the light spot should be positioned where the maximum intensity of the spectrum is observed. This ensures that the coupling of light occurs, and the peaks are more defined. 


\subsubsection{Peak broadening matching}

The spectrum for each selected wavelength in Table 1 is collected. The incident light spot is positioned so that the maximum intensity is observed. Due to the lack of instrument precision, the incident angles have slight variations from the calculations. The calculated broadening of the peaks is matched for each of the incident angles. These results are plotted in Figure 25, Figure 26, Figure 27, Figure 28, and Figure 29 for the different incident angles.

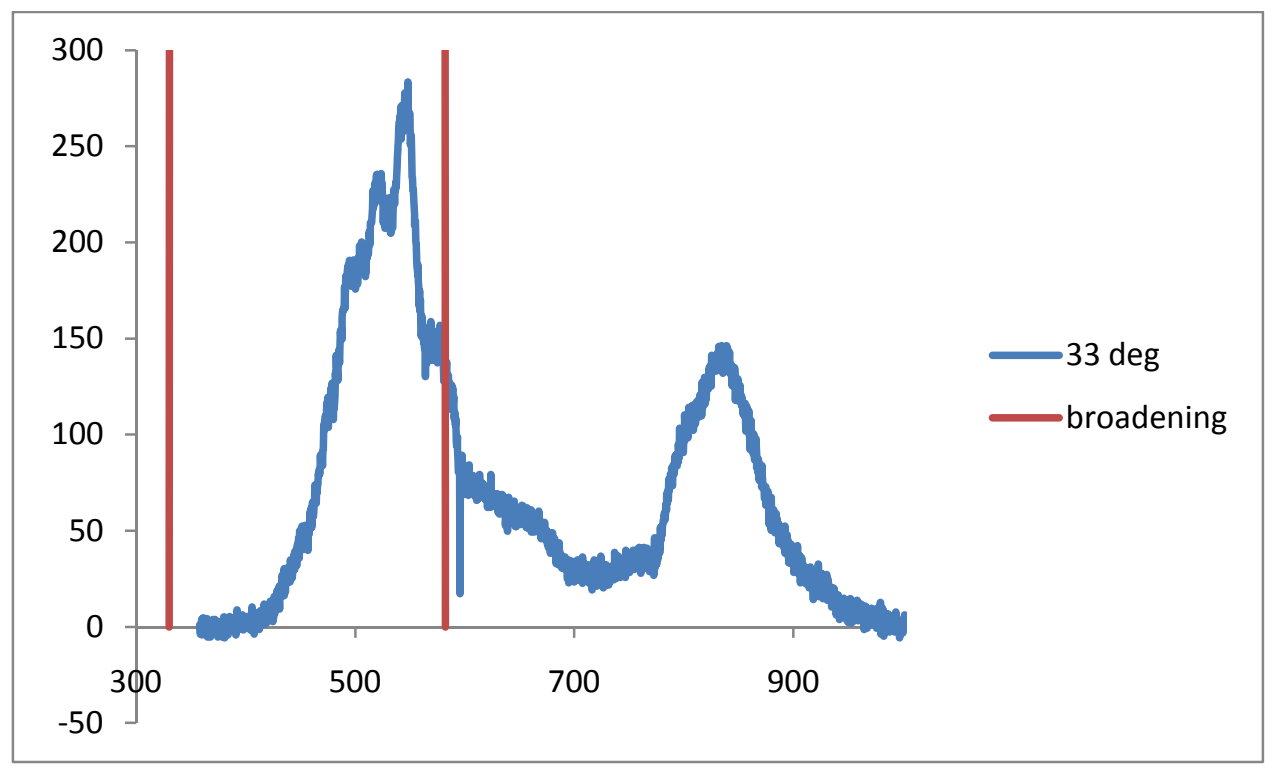

Figure 25 Peak broadening matching with incident angle $33^{\circ}$. 


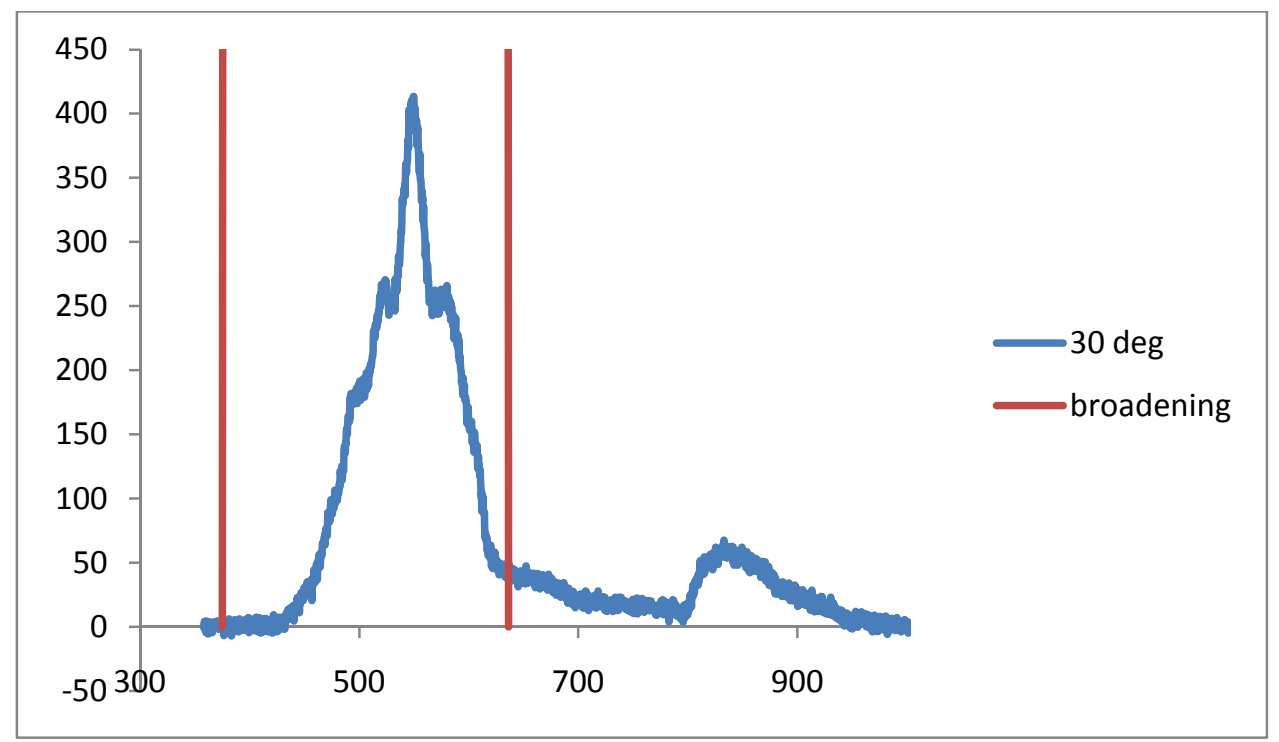

Figure 26 Peak broadening matching with incident angle $30^{\circ}$.

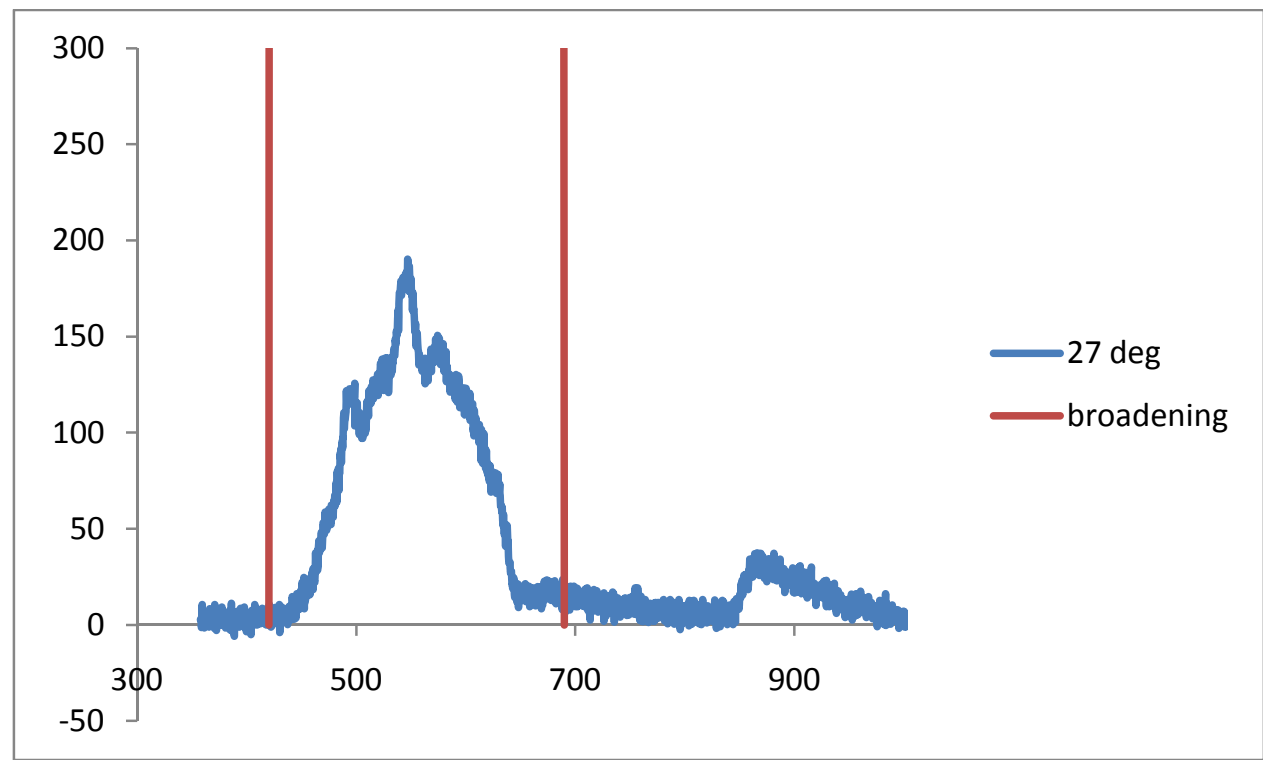

Figure 27 Peak broadening matching with incident angle $27^{\circ}$. 


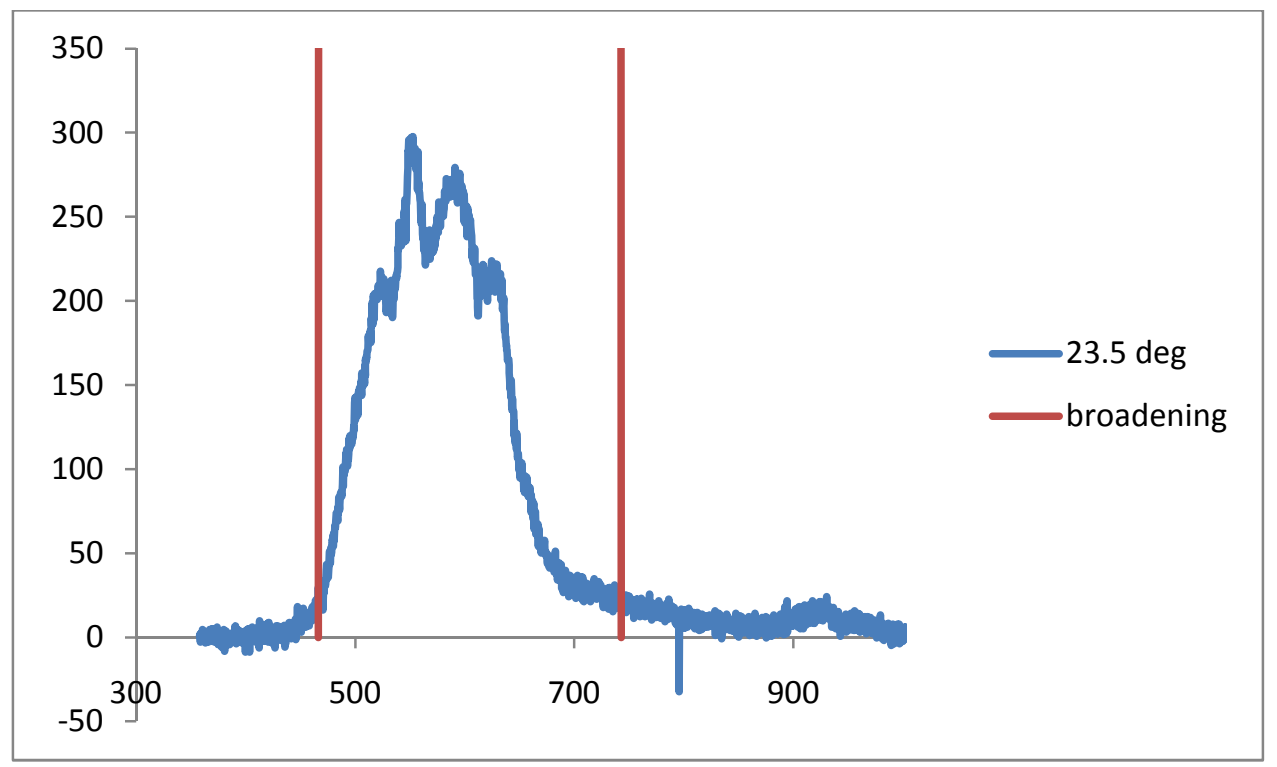

Figure 28 Peak broadening matching with incident angle $23.5^{\circ}$.

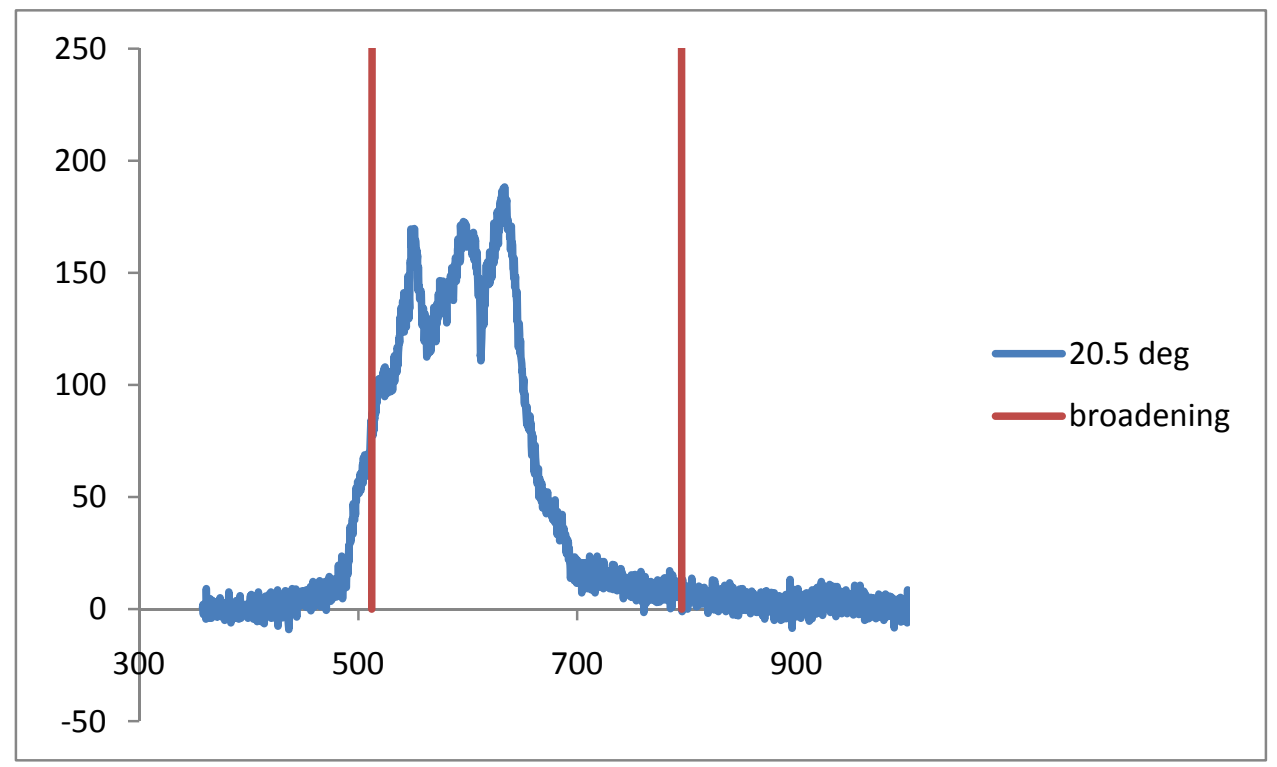

Figure 29 Peak broadening matching with incident angle $20.5^{\circ}$.

The light source does not provide enough intensity at high frequencies and has a cutoff at around 400nm. Therefore, the experimental results do not match well with the 
calculations at higher frequencies, especially for $33^{\circ}$ and $30^{\circ}$. Except for the higher frequency region, the calculation shows a good match with the experimental results.

\subsubsection{Spectra analysis}

The spectra for each incident angle is plotted and compared in Figure 30. There are multiple peaks for each spectrum which are shown in the colors in the optical images. As the incident angle becomes larger, there is also another peak rising in the near infra-red region which has not yet been explained. A thorough computational modeling of the waveguide structure may be able to explain the origin of this peak.

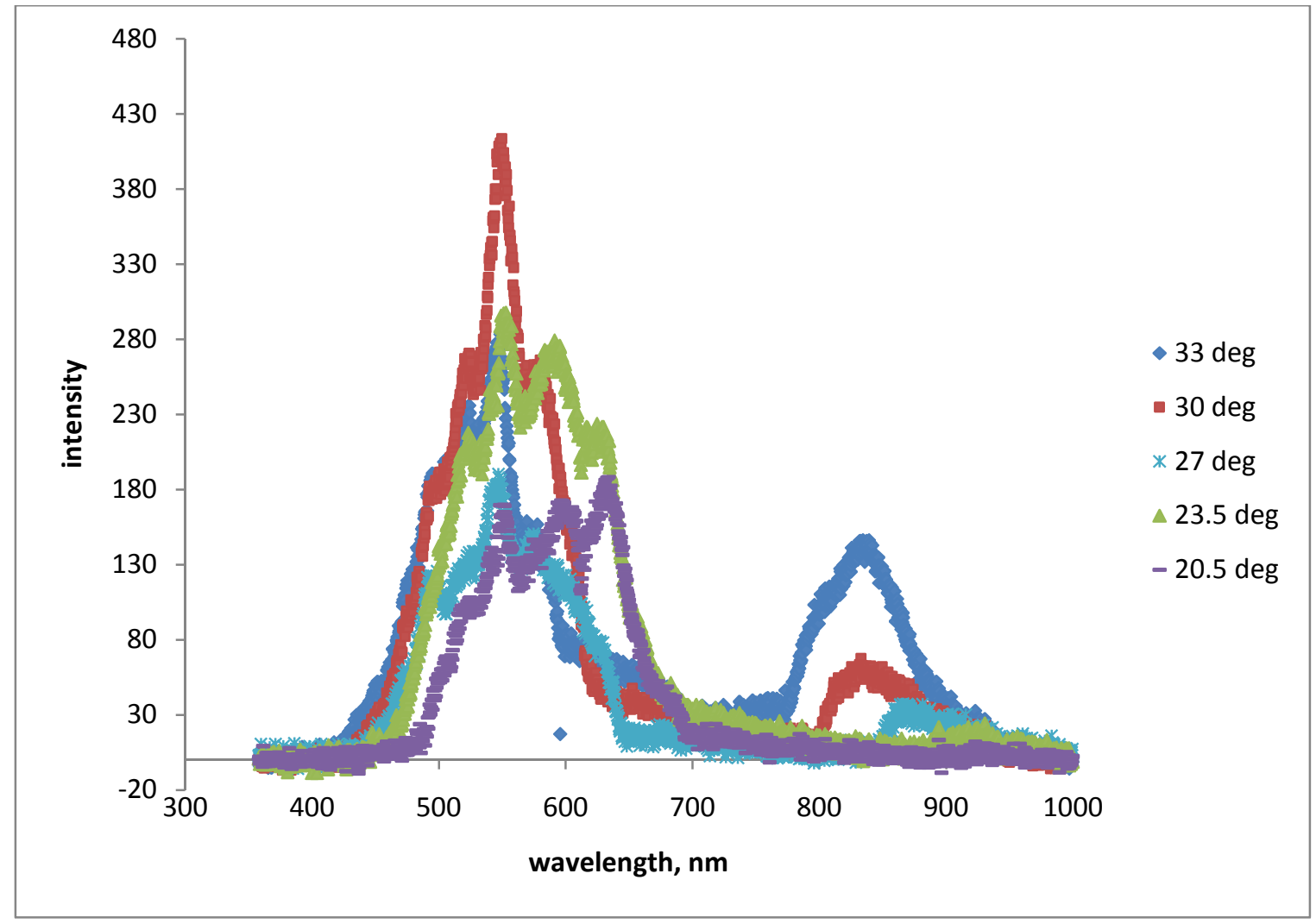

Figure 30 Comparison of the spectra between different incident angles. 
Optical images are taken for each spectrum and are shown in Figure 31.

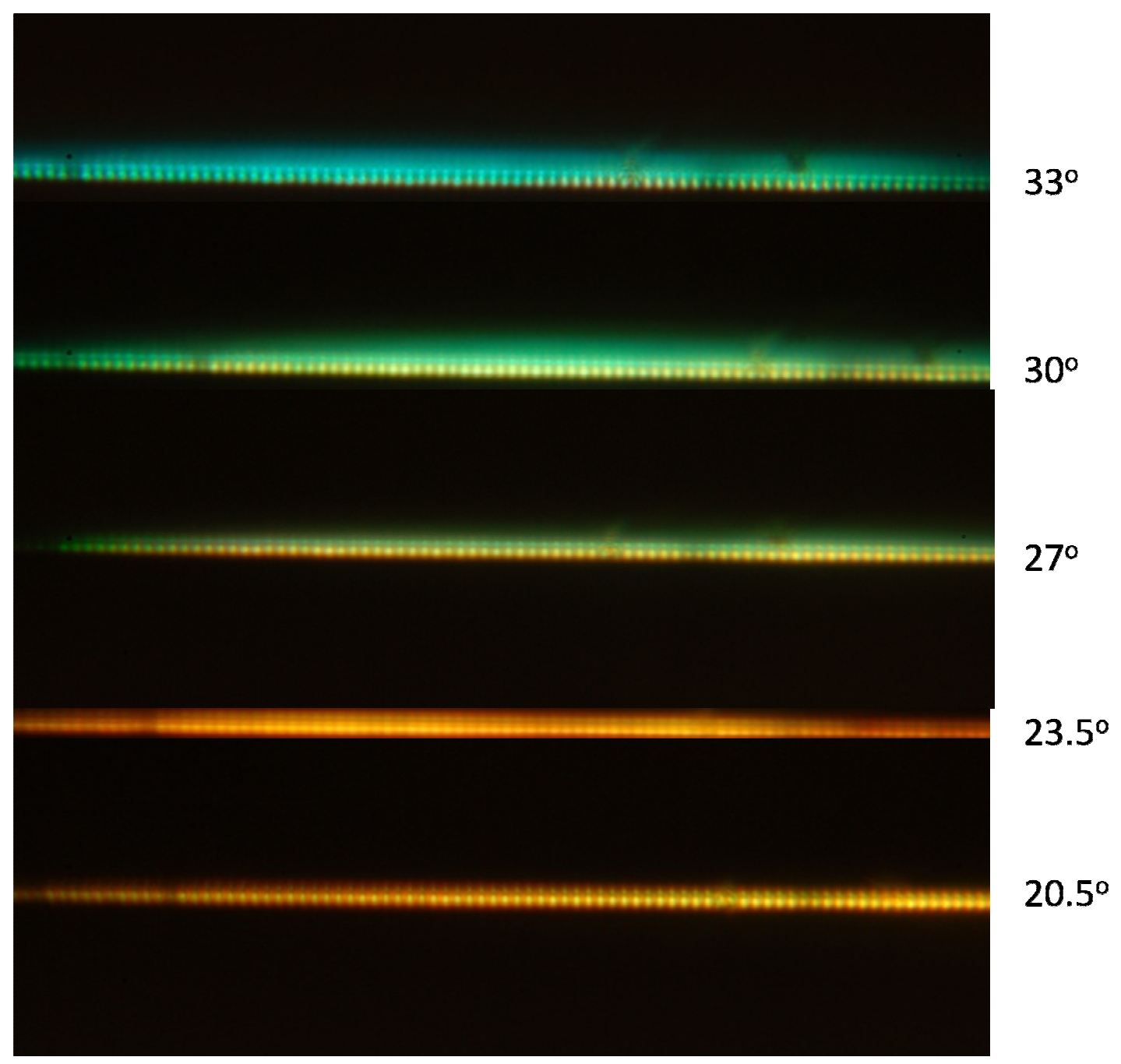

Figure 31 Optical images of the guided light with incident angles of $33^{\circ}(\sim 450 \mathrm{~nm}), 30^{\circ}(\sim 500 \mathrm{~nm})$, $27^{\circ}(\sim 550 \mathrm{~nm}), 23.5^{\circ}(\sim 600 \mathrm{~nm})$, and $20.5^{\circ}(\sim 650 \mathrm{~nm})$

Due to the complexity of the wave interactions in the waveguide structure and the presence of multi-peaks, the analysis of the spectra from different incident angles is first simplified. The peak in the near infra-red range is ignored. As opposed to multiple peaks, each spectrum is treated as a single peak. A Lorentz fitting is made to each spectrum using the Lorentz fitting function in Origin@ software from OriginLab@. ${ }^{17}$ Figure 32 shows the 
results of the fitting from the software. The $\mathrm{x}$-axis is the wavelength in nanometers, and $y-$ axis is the intensity.
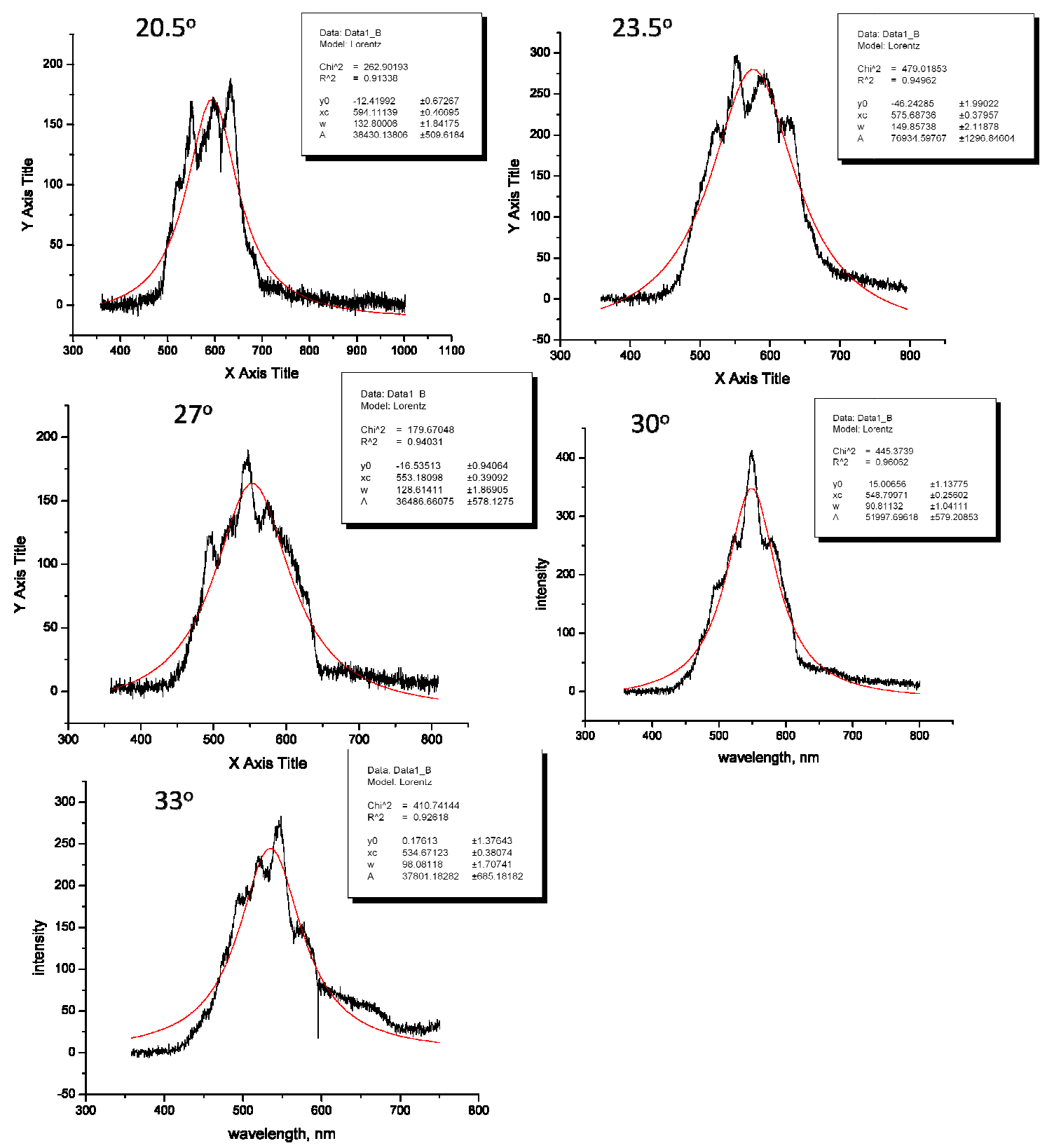

Figure 32 Lorentz fitting for each spectrum using Origin(c) software. 
The peak position in nanometer is determined from the fitting for each spectrum.

These data are presented with the calculated wavelengths in Table 2.

Table 2 Peak position and calculation for each spectrum.

\begin{tabular}{|c|c|c|}
\hline incident angle, deg & peak position & calculated wavelength \\
\hline 33 & 534.67 & 455.4 \\
\hline 30 & 548.8 & 500 \\
\hline 27 & 553.18 & 546 \\
\hline 23.5 & 575.69 & 601.3 \\
\hline 20.5 & 594.11 & 649.8 \\
\hline
\end{tabular}

To analyze the data, the diffraction equation is first manipulated: $\lambda=\frac{d}{m} \sin \theta+$ $\frac{d}{m} \sin \left(-90^{\circ}\right)$. Here $\mathrm{m}$ is -1 due to diffraction on the opposite side of the incident light.

Plotting $\lambda$ v.s. $\sin \theta$ gives a slope of $d / m$ and intercepts at $d / m$. This result is compared to the theoretical calculation shown in Figure 33.

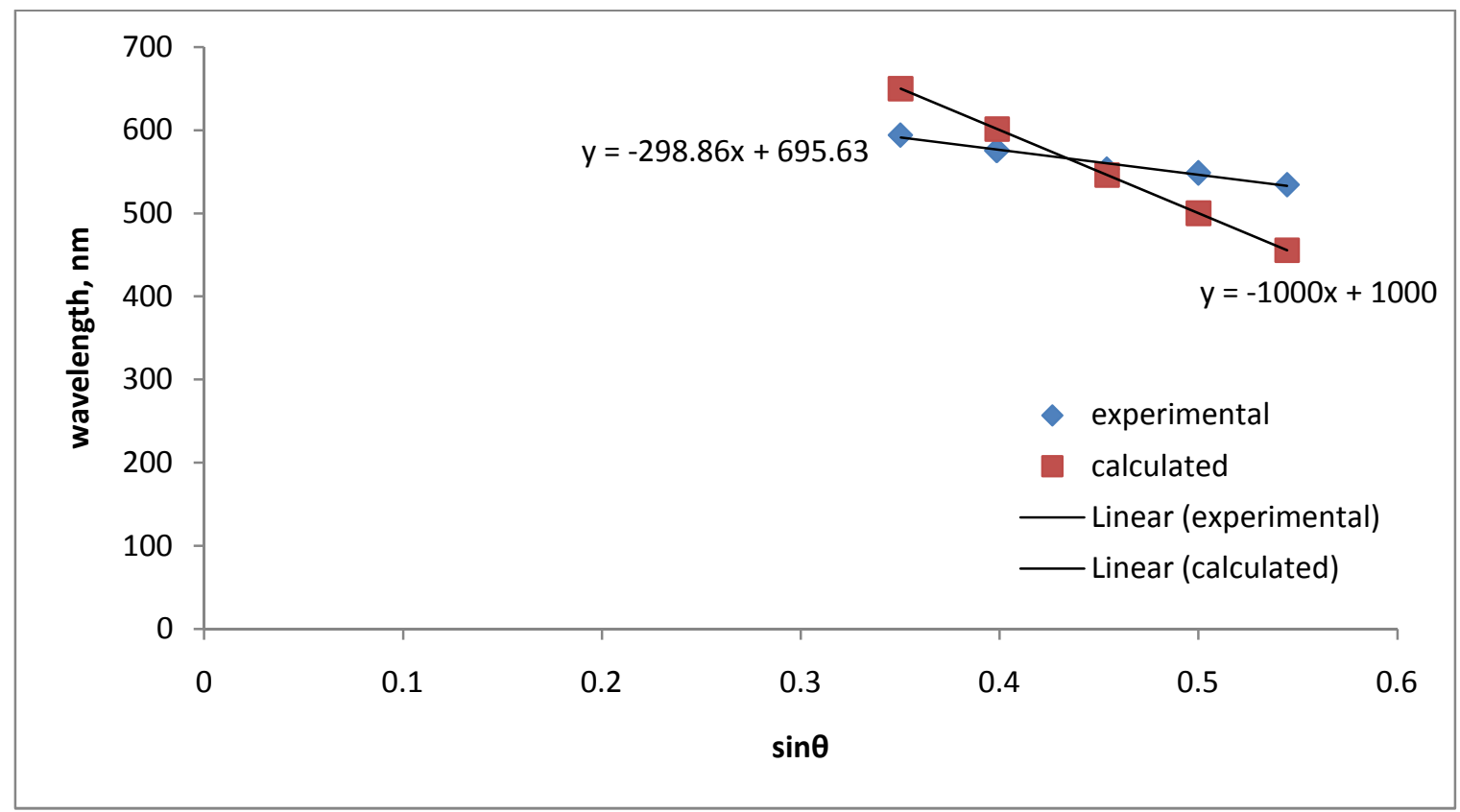

Figure 33 Comparison between the theoretical and experimental results of the peak positions. 
The experimental data shows a shift from the theoretical calculations. One possible explanation for this behavior is that the experimental data was simplified as one single peak rather than considering individual peaks. Another possibility is that of multi-modes inside the $2.5 \mu \mathrm{m}$ PU bars. ${ }^{2}$ Calculations can be done for the multiple modes of light inside the waveguide. These might be able to explain the multiple peaks observed in the spectra.

The spectra for each different angle are then normalized to if there is any shift of each spectrum. The normalization is done by dividing the spectra by the spectrum of the white light. The normalized spectra are shown in Figure 34. The spectrum shifts to lower wavelengths when the angle of incidence is increased. In addition, as the angle increases, the peak at near infra-red region starts to show. The long wavelength limit of the detector is at $1000 \mathrm{~nm}$; therefore, the presence of a peak in near infra-red region at lower incident angles cannot yet be determined.

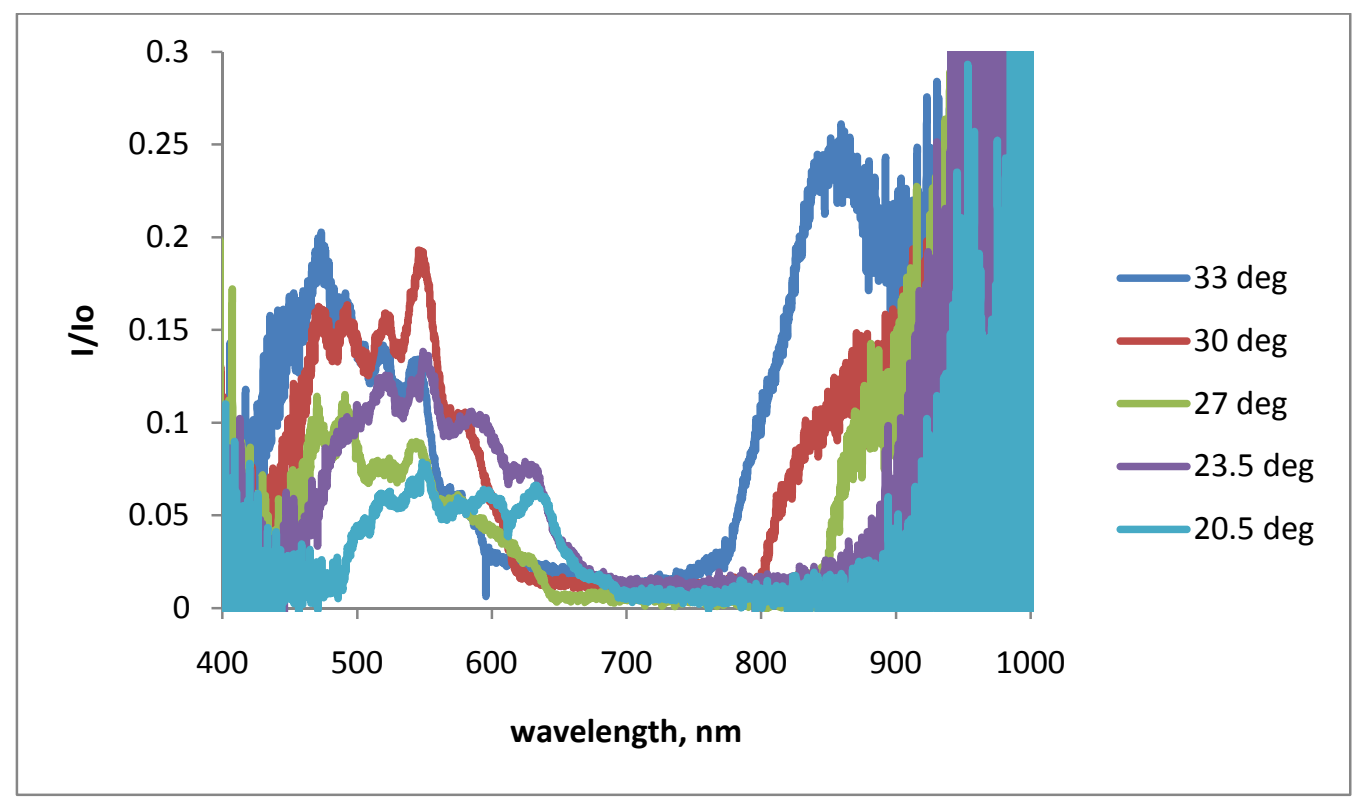

Figure 34 Normalized spectra to the white light source at different incident angles. 


\section{CONCLUSION}

Although the polymer waveguide structure had been previously established, this work extends it further using an external light source. The waveguide structure consists of light guiding $2.5 \mu \mathrm{m}$ periodicity polyurethane rods and a coupler region where $1 \mu \mathrm{m}$ and $2.5 \mu \mathrm{m}$ patterns overlap perpendicularly. Two-polymer micro-transfer molding is a well-developed fabrication technique used to make polymeric multi-layer photonic crystals. With slight changes in procedural details, a waveguide structure with $1 \mu \mathrm{m}$ on top of $2.5 \mu \mathrm{m}$ pattern was demonstrated and fabricated for characterization using $2 \mathrm{P} \mu \mathrm{TM}$.

Characterization was first done with a helium-neon laser source. Theoretical calculations are done to predict the diffraction behavior of the coupler. It is found that only the first order diffraction occurs while any higher orders do not exist with the wavelength of the laser $(632.8 \mathrm{~nm})$. Using a normal incident, the diffraction angle of the first order was calculated and experimentally determined. The experimental diffraction angle is determined to be $38.7^{\circ}$ which coincides well with the calculation of $39.3^{\circ}$. An optical image of the guided first order diffraction light was also taken by a CCD digital camera.

Characterization was completed with a white light source. The white light source provides a range of wavelengths for characterization. The results from using white light was more complex as different wavelengths are coupled into the waveguide with different incident angles. Basic calculations were done using the diffraction grating equation to predict the wavelengths of the guided light with different incident angles. For the waveguide structure used, only first order diffraction is guided. Any higher orders of diffraction either do not overlap with the first order or they do not exist. As a result of the objective lens used 
to focus the dispersed white light, peak broadening occurs for each selected incident angle. The ranges of the broadening was calculated. The experimental results of the peak broadening correspond well with the calculations. Although there seems to have multiple peaks present in the spectra for each incident angle, they were simplified as a single peak for analysis. A Lorentz fitting was done for each spectrum to find the peak position. The diffraction grating equation was altered, and $\lambda$ vs. $\sin \theta$ was plotted for each peak and was compared to theoretical calculations. However, this results in a shift of the experimental from the calculation data. 


\section{FUTURE WORK}

There are some enhancements that can be done in the experiment using the white light source. The precision of the rotating sample stage could be improved to more closely match the calculated incident angles. This can be done by using a more precise and better controlled graduation on the rotating stage and optical alignment of the sample plane and incident light. The use of the objective lens causes the peak broadening. More optics or changing to a lower magnification lens could be used to correct the unparallel incident beam with minimal broadening. Since the analysis does not take the multiple peaks into account and treats the spectrum as one peak, mode calculations of the guided light in the PU bars should be done in order to determine whether different modes give rise to the multiple peaks in the spectra. 


\section{BIBLIOGRAPHY}

${ }^{1}$ W. J. Lee, S. H. Hwang, J. W. Lim, B. S. Rho. "Polymeric waveguide film with embedded mirror for multilayer optical circuits.” IEEE Photonics Technology Letters Vol. 21. No. 1. (January 1, 2009). P12

${ }^{2}$ Lee, Jae-Hwang; Ye, Zhuo; Constant, Kristen; Ho, Kai-Ming. “Tailorable polymer waveguides for miniaturized bio-photonic devices via two-polymer microtransfer molding." Proceedings of SPIE-The International Society for Optical Engineering (2006), 6327(Nanoengineering: Fabrication, Properties, Optics, and Devices III), 63270J/1-63270J/8. Dylewicz, R.; Patela, S.; Paszkiewicz, R.; Tlaczala, M.; Bartkiewicz, S.; Miniewicz, A. ${ }^{3}$ R. E. Kunz. "Miniature integrated optical modules for chemical and biochemical sensing." Sensors and Actuators. B 38-39 (2007). P13-28.

${ }^{4}$ D.Roger, B. Christoph, B. Rene, D. Laurent, G. Max, H. Regis, H. Folkert, L. Tobias, M. Thomas, O. Stefano, S. Mauro, O. B. Jan.“ Polymer-waveguide-based board-level optical interconnect technology for datacom applications.' IEEE Transactions on Advanced Packaging (2008), 31(4), P759-767.

5 J. Vorosa,, J.J. Ramsden, G. Csucs, I. Szendro, S.M. De Paul, M. Textor, N.D. Spencer. “Optical grating coupler biosensors.” Biomaterials 23 (2002). P3699-3710. ${ }^{6}$ Christopher Palmer, Erwin Loewen(first edition). "Diffraction grating handbook." New York: Newport Corporation, 2005. $6^{\text {th }}$ Edition. http://gratings.newport.com/information/handbook/cover.asp

${ }^{7}$ M. C. Hutley. "Diffraction Gratings.” New York: Academic Press Inc., 1982. 
${ }^{8}$ Lee, Jae-Hwang; Kim, Chang-Hwan; Ho, Kai-Ming; Constant, Kristen. “Two-polymer microtransfer molding for highly layered microstructures." Advanced Materials (Weinheim, Germany) (2005), 17(20), 2481-2485.

${ }^{9}$ Dylewicz, R.; Patela, S.; Paszkiewicz, R.; Tlaczala, M.; Bartkiewicz, S.; Miniewicz, A. "Holographic lithography for grating coupler fabrication in gallium nitride grown on sapphire substrate.” International Students and Young Scientists Workshop "Photonics and Microsystems”, Proceedings, Dresden, Germany, July 7-8, 2005 (2005), 21-25.

${ }^{10}$ W. D. Callister, Jr. "Materials Science and Engineering An Introduction.” New York: John Wiley \& Sons, Inc., 2000. $5^{\text {th }}$ Edition.

${ }^{11}$ G. -L. Bona, R. Germann, B. J. Offrein. "SiON high-refractive-index waveguide and planar lightwave circuits.” IBM Journal of Research \& Development; Mar/May2003, Vol. 47 Issue $2 / 3, \mathrm{p} 239$

${ }^{12}$ S. K. J. Al-Ani, J. Beynon. "The refractive index of thin solid films." The Institute of Physics, Northern Ireland. Phys Educ. 20 1985, P274

${ }^{13}$ Summers Optical. "UV Curing." A Division of EMS Acquisition Corp. http://www.optical-cement.com/cements/cements/uv.html\#anchor669157. Jul. 292009. 14 “USB2000+UV-VIS and USB2000+VIS-NIR Miniature Fiber Optic Spectrometers.” Ocean Optics Inc., 2007. http://www.oceanoptics.com/products/usb2000+precon.asp. August 02, 209.

${ }^{15}$ H. D. Young, R. A. Freedman, T.R. Sandin (contributing), A. L. Ford (contributing). “University Physics.” Addison Wesley Longman, Inc. 2000. Volume 2, $10^{\text {th }}$ edition. 
16 “The Electromagnetic Spectrum: Visible Light Waves." National Aeronautics and Space Administration. March 27, 2007. http://science.hq.nasa.gov/kids/imagers/ems/visible.html. July 31, 2009.

17 “OriginLab Data Analysis and Graphing Software.” OriginLab@. http://www.originlab.com/. August 02, 2009. 